HD-THEP-01-001

IEM-FT-209/01

IFT-UAM/CSIC-01-01

hep-ph/0101249

\title{
Bubble Walls, CP Violation and Electroweak Baryogenesis in the MSSM
}

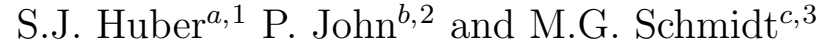 \\ ${ }^{a}$ Bartol Research Institute, 217 Sharp Lab, Newark, DE 19716, USA \\ ${ }^{b}$ Instituto de Estructura de la Materia (CSIC), Serrano 123, 28006 Madrid, \\ Spain \\ ${ }^{c}$ Institut für Theoretische Physik, Philosophenweg 16, D-69120 Heidelberg, \\ Germany
}

\begin{abstract}
We discuss the generation of the baryon asymmetry by a strong first order electroweak phase transition in the early universe, particularly in the context of the MSSM. This requires a thorough numerical treatment of the bubble wall profile in the case of two Higgs fields. CP violating complex particle masses varying with the Higgs field in the wall are essential. Since in the MSSM there is no indication of spontaneous $\mathrm{CP}$ violation around the critical temperature (contrary to the NMSSM) we have to rely on standard explicit CP violation. Using the WKB approximation for particles in the plasma we are led to Boltzmann transport equations for the difference of left-handed particles and their $\mathrm{CP}$ conjugates. This asymmetry is finally transformed into a baryon asymmetry by out of equilibrium sphaleron transitions in the symmetric phase. We solve the transport equations and find a baryon asymmetry depending mostly on the $\mathrm{CP}$ violating phases and the wall velocity.
\end{abstract}

\footnotetext{
${ }^{1}$ shuber@bartol.udel.edu

2john@makoki.iem.csic.es

${ }^{3}$ m.g.schmidt@thphys.uni-heidelberg.de
} 


\section{Introduction}

The electroweak interactions violate baryon number. There is also a source of $\mathrm{CP}$ violation in the Standard Model (SM), and possibly even stronger ones in models beyond the SM, like supersymmetric theories. Thus it is very tempting to expect that these theories which are presently under stringent experimental tests allow to explain the creation of a baryon asymmetry in the early universe. However, in thermal equilibrium a baryon asymmetry $(B+L \neq 0)$ is reduced by the electroweak interactions (conserving $B-L$ ). If there are no $B-L$ violating interactions at the GUT scale creating $B-L \neq 0$, (as e.g. in the recently discussed "leptogenesis" models [1]) one needs a strong deviation from equilibrium to create a baryon asymmetry at the electroweak scale and fast freeze out in the subsequent quasi equilibrium. Thus a strong first order phase transition with bubble nucleation is needed. However, combined analytical and lattice based work in the last years has made sure that such a phase transition is not present in the SM; actually there is no phase transition at all for Higgs masses beyond about the $W$-boson mass [2]. Thus, one has to inspect variants of the SM. Supersymmetric models are the most attractive ones. In particular the Minimal Supersymmetric Extension (MSSM) closest to the SM is investigated most intensively in experiments. With a light stop superpartner of the right handed top having a mass some GeV below the top mass, one can increase the $\phi^{3}$-term in the effective thermal Higgs potential leading to a strong first order phase transition [3 [7].

In order to create a baryon asymmetry we first want to generate a chiral asymmetry in the hot phase in front of the bubble which is then transformed into a baryon asymmetry through hot electroweak sphalerons. For this we need CP violation in the Higgs field bubble wall or some CP violation in the hot plasma disturbed by the wall. As consequence, sources are induced in the transport equations. In the MSSM the chargino and the stop mass matrices contain explicitly CP violating phases. The Higgs Lagrangian can obtain CP violating loop effects but we will also discuss in some detail the attractive possibility of a spontaneously generated "transitional" CP violation just in the bubble wall around the critical temperature [8 14]. Therefore general methods for the calculation of multi-scalar bubble walls are necessary. In Section 2 we derive the relevant equations of motion, including CP violation, and present in Section 3 a method to solve them in general. In Section 4 we present their applications to the considered supersymmetric models. 
Further on, in Section 5 we will study the transport phenomena close to the bubble wall in the quasi classical particle "WKB"-approximation 15 18. Thus we neglect soft boson classical field effects, which for example may play a role for the friction due a gauge field in the hot plasma [19], as well as off-shell memory effects to be discussed in the framework of Quantum-Boltzmann equations 2023]. The stationary velocity of the bubble wall is very important for the finally created baryon asymmetry. It is related to the friction term in the transport equations. This has been treated in ref. 24] and recently in 25] pointing to a very small velocity in the MSSM which, in general, is quite effective for producing an asymmetry. It turns out that it might even be too small for a maximum exploit [26]. In ref. [27] a class of supersymmetric models with an additional gauge singlet has been worked out. These allow for a strong first order phase transition already at tree level. In our discussing of wall profiles and CP violation we will also include this interesting case. Concerning the transport equations in the WKB approximation we can return from the calculation in ref. [28] including the singlet field to a critical discussion of the MSSM case which was already worked out in ref. 16] before and reconsidered recently [18. Finally we give numerical results for the baryon asymmetry in the given framework.

\section{Field Equations and CP Violation}

For our purposes it is sufficient to consider the one-loop corrected effective Higgs potential $V_{T}$ with thermal masses. We use the standard notation for the MSSM [29]. In general, there are CP violating phases between the Higgs field moduli $h_{1}$ and $h_{2}$. Due to gauge invariance, the effective Higgs potential only depends on one combination $\theta=\theta_{1}+\theta_{2}$ of the phases,

$$
V_{T}\left(H_{1}, H_{2}\right)=V_{T}\left(h_{1}, h_{2}, \theta\right)
$$

Defining

$$
\bar{\theta}=\theta_{1}-\theta_{2}
$$

we can rewrite the kinetic terms for the Higgs bosons in the Lagrangian density

$$
\mathcal{L}_{k i n}=\frac{1}{2} \partial_{\mu} h_{1} \partial^{\mu} h_{1}+\frac{1}{2} \partial_{\mu} h_{2} \partial^{\mu} h_{2}+\frac{h_{1}^{2}+h_{2}^{2}}{8}\left(\partial_{\mu} \theta \partial^{\mu} \theta+\partial_{\mu} \bar{\theta} \partial^{\mu} \bar{\theta}\right)+\frac{h_{1}^{2}-h_{2}^{2}}{4} \partial_{\mu} \theta \partial^{\mu} \bar{\theta} .
$$


Since $\partial_{\bar{\theta}} V_{T}=0$ the Euler-Lagrange equation for $\bar{\theta}$ is

$$
\left(h_{1}^{2}+h_{2}^{2}\right) \partial^{\mu} \bar{\theta}+\left(h_{1}^{2}-h_{2}^{2}\right) \partial^{\mu} \theta=C_{0}
$$

which minimizes the energy for $C_{0}=0$.

After elimination of $\partial_{\mu} \bar{\theta}(2.4)$, the equations of motion for $h_{1}, h_{2}$ and $\theta$ read

$$
\begin{aligned}
\partial_{\mu} \partial^{\mu} h_{1}+\frac{h_{1} h_{2}^{4}}{\left(h_{1}^{2}+h_{2}^{2}\right)^{2}} \partial_{\mu} \theta \partial^{\mu} \theta+\frac{\partial}{\partial h_{1}} V_{T} & =0, \\
\partial_{\mu} \partial^{\mu} h_{2}+\frac{h_{2} h_{1}^{4}}{\left(h_{1}^{2}+h_{2}^{2}\right)^{2}} \partial_{\mu} \theta \partial^{\mu} \theta+\frac{\partial}{\partial h_{2}} V_{T} & =0, \\
\partial_{\mu}\left[\frac{h_{1}^{2} h_{2}^{2}}{h_{1}^{2}+h_{2}^{2}} \partial^{\mu} \theta\right]+\frac{\partial}{\partial \theta} V_{T} & =0 .
\end{aligned}
$$

Two limits are important. The first limit corresponds to a radially symmetric spatial solution of

$$
\frac{\partial^{2} h_{i}}{\partial r^{2}}-\frac{2}{r} \frac{\partial h_{i}}{\partial r}-\frac{\partial V_{T}\left(h_{i}\right)}{\partial h_{i}}=0
$$

(without $\mathrm{CP}$ phase $\theta$ ). The solution describes the initial state of nucleating bubbles, the "critical bubble". Eqs. (2.8) are also to be solved for the determination of the tunneling probability [30 32].

Second, constraining equations (2.5)-(2.7) to a stationary wall ("domain wall") moving with velocity $v_{w}$ we are left with just one direction $x=z-v_{w} t$ at late time $t$ perpendicular to the wall. This is the more important period for baryogenesis. With the assumption of stationarity and almost planar bubble walls the equations (2.5)-(2.7) reduce to

$$
\begin{array}{r}
h_{1}^{\prime \prime}+\frac{h_{1} h_{2}^{4}}{\left(h_{1}^{2}+h_{2}^{2}\right)^{2}} \theta^{\prime \prime}-\frac{\partial}{\partial h_{1}} V_{T}=0, \\
h_{2}^{\prime \prime}+\frac{h_{2} h_{1}^{4}}{\left(h_{1}^{2}+h_{2}^{2}\right)^{2}} \theta^{\prime \prime}-\frac{\partial}{\partial h_{2}} V_{T}=0, \\
\partial_{x}\left[\frac{h_{1}^{2} h_{2}^{2}}{h_{1}^{2}+h_{2}^{2}} \theta^{\prime}\right]-\frac{\partial}{\partial \theta} V_{T}=0,
\end{array}
$$

where the prime denotes $\partial_{x} . V_{T}$ is deformed by the plasma (moving in the wall frame) friction and has degenerate minima in the stationary case like $V_{T=T_{c}}$. We roughly identify the deformed $V_{T}$ with $V_{T=T_{c}}$. 
For the NMSSM singlet field $S$ it is more convenient [13, 28] to divide it up into real and imaginary components,

$$
S=n+i c
$$

implicitly introducing a phase variable $\theta_{S}=\arctan (n / c)$. The equations of motion for $n$ and $c$ are of Klein-Gordon type

$$
\begin{aligned}
\partial_{\mu} \partial^{\mu} n+\frac{\partial}{\partial n} V_{T} & =0, \\
\partial_{\mu} \partial^{\mu} c+\frac{\partial}{\partial c} V_{T} & =0,
\end{aligned}
$$

where $V_{T}$ is the NMSSM potential [28].

In Sec. 4.3 we will see that especially the variations of the $\mathrm{CP}$ violating quantities $\theta$ and $c$ in the bubble wall play a very important role in the generation of the baryon asymmetry. But first we will discuss more generally how these equations may be solved.

\section{How To Find Bubble Wall Profiles}

The first order phase transition is mediated by expanding bubbles. In order to compute the baryon asymmetry, we should follow the history of bubbles from the moment of nucleation, until the time when the broken phase fills the Universe. After nucleation, there is in general a long period of stationary growth. Nucleation is characterized by time dependent solutions to the full equations of motion in a background of in general non-trivial profiles for temperature, velocity and chemical potential. In the SM such solutions were investigated in [33]. We will not consider the full problem here but focus on the profiles of stationarily expanding almost planar bubble walls at the critical temperature $T_{c}$ which interpolate between two minima of the effective potential. This case is more interesting case for baryogenesis. In case of friction with the background plasma the corresponding temperature is the nucleation temperature $T_{n}$ [25].

For the bubble wall profiles in the MSSM without CP violation there exist numerical approaches to solve the problem of critical bubbles with two Higgs fields in [34] and more general in [35]. In [10], the CP profile has been investigated in the background of a fixed Higgs profile. We will now discuss methods which enable us to find profiles in more general scenarios as shown in Sections 4.1 and 4.2 as well as in [13, 25]. 
The usual method for the SM case with only one Higgs field is to solve the corresponding single equation of type (2.13) numerically by "turning around" the effective potential $V_{T}$ and dealing $x$ for a time $t$. Then the problem can be regarded as an initial value problem for the inverted potential $-V_{T}$. Usually an "overshooting-undershooting" procedure can be applied. This works well since there is only one direction in field space. Moreover, it can be implemented quite simply by the standard Runge-Kutta-method.

The situation is completely different once there are additional directions in field space. Again one can consider the analogous mechanical problem with the turned around potential. Neglecting first derivatives which act like friction on the potential, the initial value problem is equivalent to a mass point rolling from the top of one hill (first minimum) along the ridge such that it comes to rest on the top of the second hill (second minimum). Small changes in the initial conditions lead to a completely different shape of the solution. In general it is not possible to know the initial conditions with sufficient accuracy to find the desired solution. Hence we have to device another method. Here we use the method of minimization of the functional of the squared equations of motion.

A stationary problem for big bubbles can be reduced to a problem in one spatial coordinate $x=z-v_{w} t$ for a constant wall velocity $v_{w}$. A constant velocity is caused by friction with the background plasma which ensures that the minima are degenerate [24, 25, 30, 32, 36].

To solve Eqs. (2.9)-(2.11) one has to find field configurations for which

$$
S=\int_{-\infty}^{+\infty} d x\left[E_{1}^{2}(x)+E_{2}^{2}(x)+E_{3}^{2}(x)\right]=0 .
$$

This is achieved by searching for the absolute minimum of $S$. Here $E_{1}(x), E_{2}(x)$ and $E_{3}(x)$ are the left hand sides of Eqs. (2.9), (2.10), and (2.11), respectively. For the NMSSM the extension is straightforward

$$
S=\int_{-\infty}^{+\infty} d x\left[E_{1}^{2}(x)+E_{2}^{2}(x)+E_{3}^{2}(x)+E_{4}^{2}(x)+E_{5}^{2}(x)\right],
$$

where $E_{4}(x)$ and $E_{5}(x)$ are the squares of stationary versions of the l.h.s. of Eqs. (2.13) and (2.14):

$$
\begin{gathered}
E_{4}(x)=n^{\prime \prime}-\frac{\partial V_{T}}{\partial n}, \\
E_{5}(x)=c^{\prime \prime}-\frac{\partial V_{T}}{\partial c} .
\end{gathered}
$$


This minimization method has also been successfully used in [34 for the critical bubble. The approach of [37] is in principle also a minimization procedure and therefore the following considerations are also applicable. The method of [7, 355 deals directly with the saddle point. Using the minimization method we have to solve a boundary value problem instead of an initial value problem. Thus we have to use an ansatz which fulfills the boundary conditions for each function for which we want to find the time development.

Our procedure works in two steps. The first crucial step is to find an ansatz which is as close as possible to the exact solution.

Step One: Ansätze In [6, 13, 34, 38] it was found that a kink is a good ansatz for the Higgs fields. We therefore use for $N$ Higgs fields

$$
\phi_{i}^{k i n k}=\frac{v_{i}}{2}\left(1+\tanh \left(\frac{x}{L_{i}}+\hat{x}_{i}\right)\right), \quad i=1 \ldots N .
$$

The $L_{i}$ and $\hat{x}_{i}$ are determined by minimizing (3.1) or (3.2) which are built up from these configurations in the Eqs. of motion like (2.13)-(2.14). This first step is cheap in terms of computer time since it is only a $N$-dimensional minimization and can be performed very fast. Minimizing with respect to a few parameters is a very successful first step since it reduces the value of (3.1) already significantly compared to a general function which only fulfills the boundary conditions. Hence the convergence is considerably improved.

The shape of the tunneling trajectory depends strongly on the CP-odd Higgs mass parameter $m_{A}$. Small values of $m_{A}$ give a larger mixing of the CP even Higgs fields and a sharper bending curve (see [38]).

Also the ridge between the the peaks turns out to be a rather fine ansatz. In general, the ridge is difficult to define but often it can be defined sufficiently in two scalar field dimensions. In a generic NMSSM case the ridge is even nearer to the solution than a superposition of two kinks. This is demonstrated in Fig. 2, where the solution and the ridge lie quite close to each other.

ridge In two scalar dimensions an approximation to the ridge often can be defined as follows: Intersect the effective potential in planes perpendicular to the straight connection between the minima and take the maxima of the intersection curves in those intersection planes as points of the ridge.

To use this in the NMSSM where we have basically 3 fields we define a new average field $h=\sqrt{h_{1}^{2}+h_{2}^{2}}$ in the $h_{1}-h_{2}$ plane. This is a good approximation for 
$h_{1}$ and $h_{2}$ which are close to a straight line, similarly to the MSSM. They can subsequently be treated as one. Then, a good approximation to the ridge can be found by applying the above construction to the effective potential in the $h-S$ space. In Sections 4.2 we will discuss examples which violate CP. In these cases the ridge cannot be defined in the described simple manner. Fortunately these example cases again permit ansätze utilizing the extended kink (3.5).

Step Two: Minimization and problems connected with it: With a preoptimized ansatz we come to the second step, the high dimensional minimization. To represent (3.1) and (3.2) numerically we discretize them over a grid. We have to discretize the space variable $x=z-v_{w} t$, the fields $\phi_{i}\left(x_{j}\right)$, the derivatives of the fields (first and second derivatives), and derivatives of explicitly given functions like the effective potential. These points have recently been discussed partially in [7, 34, 38]. We want to discuss here some forthcoming details and computational problems.

The fields are defined on a grid of points $x_{j}$ along $x$. The first derivatives turn out to be appropriately discretized by $f^{\prime}(x)=(f(x+\epsilon)-f(x-\epsilon)) / \epsilon$ and the second derivatives by $f^{\prime \prime}(x)=(f(x+\epsilon)-2 f(x)+f(x-\epsilon)) / \epsilon^{2}$. Accuracy is of order $\epsilon^{2}$ where $\epsilon$ is the grid spacing. The discretized field equations (3.1) and (3.2) can be built out of these elements and the discretized fields. Derivatives including more points may even impair the result since they require more additions and subtractions which are numerically problematic. For this problem see also [39, 40].

Minimization parameters are the values $\phi_{i}\left(x_{j}\right)$ and $\theta\left(x_{j}\right)$, respectively. With $N$ fields defined at $M$ grid points we have a $N \times M$-dimensional function to be minimized.

In our applications the minimization itself is accomplished using two different methods, "Powell's method" [39] and a "Pseudo-Langevin" method which will be discussed below. We also used the "downhill simplex" minimization method [39] for comparison with [11] which turned out to be too slow for practical use beyond a few computations.

For the discussion of the practical usage of minimization we first discuss a common problem of minimizations including numerical derivatives. In general several undesired minima occur which can be categorized as follows:

a) Some are real solutions of the equations of motion. A trivial example is $\theta(x)=0$, which always solves (2.5)-(2.7).

b) Fake minima due to the numerical representation of the functional. This is 
a common problem of discretized derivatives with finite differences.

c) Minimization of (3.1) is achieved by solving $\delta S=0$, which is only a necessary condition for solution configurations. For a squared form, $S=a^{2}$, also pseudosolutions according to $\delta a=0$ may exist in addition to the (desired) solution $a=0$. Also combined effects may occur as discussed in 38 .

Altogether this induces the problem that, starting from an ansatz which is in the vicinity of such an apparent solution, the algorithm might never converge to the desired exact solution. How can these problems be solved?

One method is related to the method of "Simulated Annealing" [39, 41]. There the trajectory through the high dimensional configuration space is given by a Langevin type equation, e.g.

$$
\frac{d q_{i}}{d \tau}=-\frac{\partial S[q]}{\partial q_{i}}+\eta_{i}(\tau),
$$

where the $q_{i}$ are the degrees of freedom of the discretized action $S[q]$ (Eq. (3.1) or (3.2) and $\eta_{i}\left(\tau_{n}\right)$ are random variables, typically Gaussian distributed. Every configuration is randomly varied to jump out of unwanted local minima. For a global progress in minimizing $S$ a small suffer loss is permitted. An algorithmic temperature is defined which regulates the amplitudes of the fluctuations. This method is commonly used in lattice calculations (e.g. [42]). Unfortunately, the action $S$ in our case is quite sensitive to randomly varying degrees of freedom through the derivatives of the configurations. A random change in the fields causes a strong change in the derivatives which increases $S$. The convergence is very poor and it turns out that this method does not solve our problem.

Writing down the discretization of (3.6) and omitting the fluctuation term we find the "Pseudo-Langevin" equation

$$
q_{i}\left(\tau_{n+1}\right)=q_{i}\left(\tau_{n}\right)-\Delta \tau \frac{\partial S[q]}{\partial q_{i}\left(\tau_{n}\right)} .
$$

Now the new time interval $\Delta \tau$ of the new Hamiltonian system is a common integration step for all degrees of freedom. The motion promoted by Eq. (3.7) is a motion without inertia through the configuration space. It converges towards a minimum of $S . \Delta \tau$ is determined through the scale of $\partial S[q] / \partial q_{i}(n)$ :

$$
\Delta \tau \sim \frac{\partial S[q]}{\partial q_{i}(n)}
$$

In order to get a stable trajectory it must be sufficiently small. Now, a magnification of $\Delta \tau$ after some time steps causes a "coherent" jump of the whole system 
out of a local minimum. This is exactly the desired behavior of the "Simulated Annealing" method. But in that case the "decoherent" variation of the configuration shifts the system configuration to a completely unrelated point, permitting almost no effort at all in minimizing $S$. Thus, the system starts again from a worse configuration. It converges extremely slowly for a considerable number of degrees of freedom. However, the coherent annealing of our Pseudo-Langevin approach allows to "jump" over local hills without completely loosing properties of the configuration. It permits a larger time step to overcome small bumps and troughs, see Fig. 11.

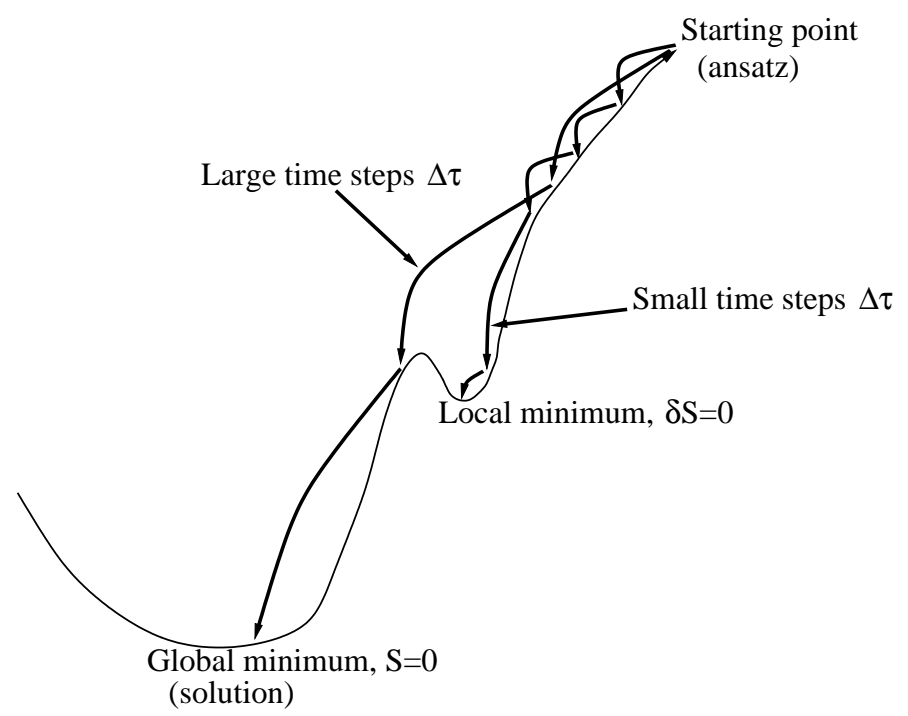

Figure 1: Pseudo-Langevin method: Large time steps allow to overcome small bumps and troughs. Small time steps or simple minimum finding routines may lead into undesired local minima.

This method is quite efficient and sometimes called "gradient descent" method. An interesting variant is also used in [7, 35] which directly deal with the saddle point configuration.

The limit of our method is reached with derivatives $\frac{\partial S[q]}{\partial q_{i}(n)}$ in (3.7) for the $q_{i}$ varying on strongly differing scales. Then the time step can not be computed appropriately for all $q_{i}$ simultaneously and the system runs over a minimum at one $q_{i}$ while another $q_{j}$ has not changed considerably at all. Such a behavior appears for large $h^{3}$-bumps in the effective potential. This may already be the case in the NMSSM for some parameter combinations. But a combination of all presented methods can also treat more complicated cases we cannot discuss here.

What can be done to avoid some of the problems and to rate the quality of a minimum found? First it is important to have an ansatz as close as possible 
to the desired solution to avoid reaching an unwanted local minimum after the time consuming second minimization step. One can also increase the number of intermediate steps in the procedure to improve the precision at the cost of computing time. Additionally we give an independent check to rate the results.

Rating of solutions As long as we have energy conservation, one can check the quality of the results easily. The relation

$$
\begin{array}{cl} 
& V\left[q_{i}\right] / T\left[q_{i}\right]=1 \\
\text { or } \quad & V\left[q_{i}\right]-T\left[q_{i}\right]=0 \quad \text { for all } x(\text { or } \tau),
\end{array}
$$

where $T\left[q_{i}\right]$ is the kinetic part of the action, must be fulfilled and one can check the deviation. With our Pseudo-Langevin approach the best solutions reach $V[q] / T[q]-1$ with a precision of order $10^{-3}$. However, such a high precision is not necessary, even for sensible quantities like $\Delta \beta$. One can construct further identities that have to be fulfilled like an extended virial theorem as in [37.

There is also a possibility for cases without energy conservation. Let us first discuss the problem of shooting along a given path in the Higgs field space because this method also gives a quality check for non energy conserving cases. Imagine that we have chosen a path for the bubble wall solution in field space. This does not mean that we have solved the equations of motion which would yield the $x$ dependence of the fields. But for a given path we can use the overshootingundershooting procedure to find the $x$-dependence. The naive method to take the potential along the path as an effectively 1-dimensional potential gives wrong results even for cases with energy conservation. It can lead to a wall thickness which differs from the real result by a factor of two or more. The reason for this is that the differential equations only contain partial derivatives. In the naive approach the effective derivative (along the path) is a combinations of the original (partial) derivatives. But it can be extended to construct a quality check of solutions by looking at each field space direction separately.

One necessary condition for reliable results is the shooting condition for the bounce solution: The "marble" rolling along an arbitrary path must come to rest at the zero minimum (symmetric phase). The field configuration $\bar{q}$ which defines the path coordinates is a combination of the scalar fields $\left(h_{1}, h_{2}, \ldots\right)$. A necessary condition for the solution configuration is

$$
\int_{x_{\text {start }}}^{x_{\text {end }}} d x \frac{\partial V[\bar{q}]}{\partial h_{k}}=0,
$$


which has to be fulfilled separately for all field directions $k$. Each configuration $h_{k}(x)$ can be projected to the chosen path $\bar{q}$ leading to configurations $\bar{q}_{k}(x), k=1 \ldots N$ for $N$ fields. Vice versa, assuming that $\bar{q}(x)$ is the exact solution to the (critical) bubble wall equations, all $\bar{q}_{k}(x)$ are then identical by construction. We can use this to construct a general check. The configuration $\bar{q}(x)$, obtained by shooting along a chosen path, may be projected to all components $h_{k}(x)$ in the field space which automatically fulfill (3.11) independently. The inverse functional $x\left[h_{k}\right]$ can subsequently be used to construct a motion configuration $\bar{q}$ along the path for every $k: \bar{q}_{k}\left(x\left[h_{k}\right]\right)$. For the solution this leads to $N$

identical configurations $\bar{q}_{k}\left(x\left[h_{k}\right]\right)$. But every pseudo solution different from the exact solution configuration leads to differences in these reconstructed pseudosolutions which rates the quality of the pseudo solution.

Concluding, the path has to be chosen that

$$
\int_{x_{\text {start }}}^{x_{\text {end }}} d x \frac{\partial V}{\partial h_{1}} \ldots=\int_{x_{\text {start }}}^{x_{\text {end }}} d x \frac{\partial V}{\partial h_{i}}=\ldots=0 \quad \forall h_{i}
$$

(shooting condition) and simultaneously the (inverted) motion along the path must be the same for every field $h_{i}$. This even works for critical bubbles where we have no energy conservation and the energy check does not work.

The methods presented here can be used to investigate the dynamics of bubble wall expansion and baryogenesis in more detail. In Section 1 we extend them to investigate $\mathrm{CP}$ violation for many parameter combinations. Our considerations and calculations were also essential to develop better perception of which approximations might be useful and appropriate to simplify calculations as done in Section 5. More applications can be found in refs. [13, 25, 27, 28]

\section{CP violation}

To calculate the actual baryon asymmetry $\mathrm{CP}$ violation as one of the Zakharov conditions becomes important. In [16, 22, 23, 43, 45] it was found that a sufficient baryon asymmetry requires $\mathrm{CP}$ violating phases as large as $\mathcal{O}\left(10^{-1}\right)$. This might lead to contradictions with experimental constraints [46] on the electric dipole moment of the neutron (EDM). It is still under discussion whether this contradiction might be resolved by special cancellations. Large phases are also allowed if the 1st and 2nd generation squarks are heavy [47. Regarding our results in the final Section 6 this becomes important again. For a more detailed discussion of this point see refs. [48] and [49]. 

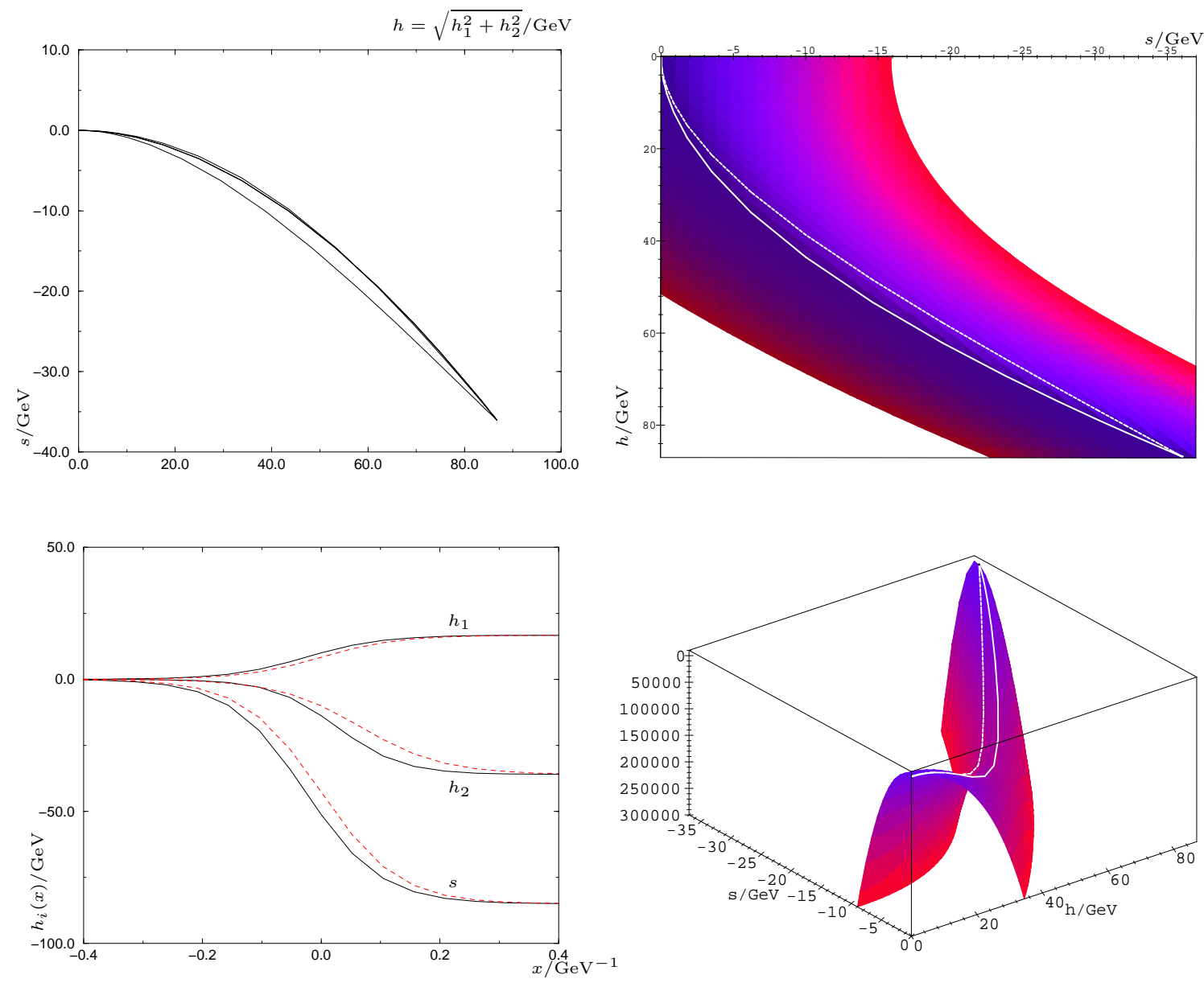

Figure 2: Solution and ansätze in the NMSSM. Left top: Fitted $\tanh (x / L+\hat{x})-$ ansatz and the ridge lying directly near the actual solution. Lower left: Shape of solution versus $x$ compared to a kink ansatz. Right: 3-dimensional views of the effective potential with solution and kink ansatz.

It is known, that in principle there may be spontaneous $\mathrm{CP}$ violation in the MSSM at zero temperature realized by a CP violating phase between the two Higgs doublets. While spontaneous $\mathrm{CP}$ violation at $T=0$ is forbidden for the 
experimental allowed parameters [8], it was suggested that it might be realized at finite temperature quite naturally [9, 50] or especially only in the bubble wall between the symmetric and the broken phase [10, 11, 51].

An interesting scenario is therefore a temperature induced $\mathrm{CP}$ violation in the bubble wall during the first order electroweak phase transition which is not restricted by any experimental bounds. Hence it may be maximal and might sufficiently support the generation of the baryon asymmetry of the Universe 17 , 52. It will be referred to as transitional CP violation in contrast to $\mathrm{CP}$ violation in the broken minimum. In the literature [9 12,50,51] the conditions for transitional CP violation were widely discussed. In Section 4.1 we will revisit this question by following the approach of ref. [13] in more detail. Since this approach is different to previous ones we compare the methods.

Explicit CP violation is introduced by complex mass parameters in the effective potential. Independently from strong experimental bounds it might be interesting to study its effects on bubble walls and whether it suffices to contribute considerably to the baryon asymmetry. In Sec. 4.2 we will analyze in detail the evolution of explicit $\mathrm{CP}$ violating phases in the bubble wall.

\subsection{Transitional CP Violation in the MSSM: Revisited}

In this section we elaborate on the systematic search of 13] for a parameter window for transitional CP violation in the MSSM. First we consider the case without explicit $\mathrm{CP}$ violating phases and check whether a $\mathrm{CP}$ conserving solution $(\theta=0, \pi)$ is a local minimum of the action. Instability in the $\theta$-direction requires

$$
m_{3}^{2}\left(h_{1}, h_{2}\right):=\left.\frac{1}{\left|h_{1} h_{2}\right|} \frac{\partial^{2} V_{T}\left(h_{1}, h_{2}, \theta\right)}{\partial \theta^{2}}\right|_{\theta=0}<0 .
$$

$m_{3}^{2}\left(h_{1}, h_{2}\right)$ is a measure for the instability at a given point in the wall. $V_{T}$ is the finite temperature effective potential including stops, charginos and neutralinos. In our convention $h_{1}$ can have either sign which permits to consider only $\theta=0$. Condition (4.1) agrees with the constraint of Lee [53] on which [8, 9, 11, 51] are based on. But Eq. (4.1) is true more generally along the whole bubble wall.

We are interested in regions where (4.1) is fulfilled. In our search we first neglect the strong experimental bounds on the Higgs mass since it might still be interesting to find a shape of such a transitional $\mathrm{CP}$ violation. The region of generically allowed parameters is an 8-dimensional "hypercube" stretched by the tree level parameters $m_{A}$ and $\tan \beta$, the stop mass parameters $m_{Q}, m_{U}$, trilinear 
couplings $A_{t}$, and $\mu$ as well as the gaugino mass parameters $M_{1}$ and $M_{2}$ in the neutralino and chargino mass eigenvalues. For each chosen parameter set we must determine the critical bubble wall profile $h_{1}(z), h_{2}(z)$ including the $\theta(z)$ field and scan the wall for points which fulfill the instability condition (4.1).

The methods for solving the (critical) bubble equations with more than one scalar field including $\theta$ were discussed in Section 3 and in [38]. However, all these methods are very time consuming and need sophisticated algorithms. Therefore we proceed in two steps. First we will restrict the possible parameter space in order to obtain a more promising parameter set permitting transitional $\mathrm{CP}$ violation. Hence we will investigate the averaged dependence on the parameters. Second, with this preparation we will search for a definite parameter set permitting the desired CP violation in the bubble wall.

A first and useful simplification is to refrain from determining the wall profile very accurately for each parameter set. Indeed, in the MSSM the solution to the equations of motion is quite close to a kink ansatz $h(z) \sim 1 / 2(1+\tanh (z / L))$, and therefore we do not need the full solution to the equations of motion. The solution can be approximated by the ridge of the negative potential $-V_{\text {eff }}$. Actually, it is even sufficient to approximate the solution in field space $h_{1,2}$ by the straight line between the minima. This approach is correct as long as there is only small CP violation which does not have a too strong back-reaction to the wall shape as could happen for large phases (see ref. [38]). If we found a candidate for transitional CP violation with a large phase, we would have to solve the whole set of equations. But for the moment we are only searching for promising parameter regions.

In order to perform a complete search for a parameter window with transitional $\mathrm{CP}$ violation in the bubble wall it is important to consider the dependences on the various parameters. At tree level we find

$$
m_{12}^{2}=-\frac{1}{2} m_{A}^{2} \sin 2 \beta
$$

Hence, the minimum in $\theta$ direction is at $\theta=0$. Thus, in order to obtain spontaneous $\mathrm{CP}$ violation the 1-loop contributions have to overcome the tree level term. Obviously large $\tan \beta$ and small $m_{A}$ are preferred. The dependence on other parameters is not quite obvious. Potentially it is possible that $m_{3}^{2}$ is negative for very small (negative) $m_{U}^{2}<<(2 \pi T)^{2}<<m_{Q}^{2}$ [12, 13].

In an analytical approach where we plot $m_{3}^{2}$ versus various parameters we always fix the remaining parameters. We find that it dominantly depends on $m_{A}$ and $\tan \beta$. All other parameters are effectively 1-loop corrections and give a 
correspondingly smaller effect. The strongest 1-loop dependence is given by $A_{t}$ and $\mu$ (see Fig. (3). We also find here that large values with opposite signs give the smallest $m_{3}^{2}$. Later we will see that the latter effect appears only to be correct in these preliminary analytical investigations.

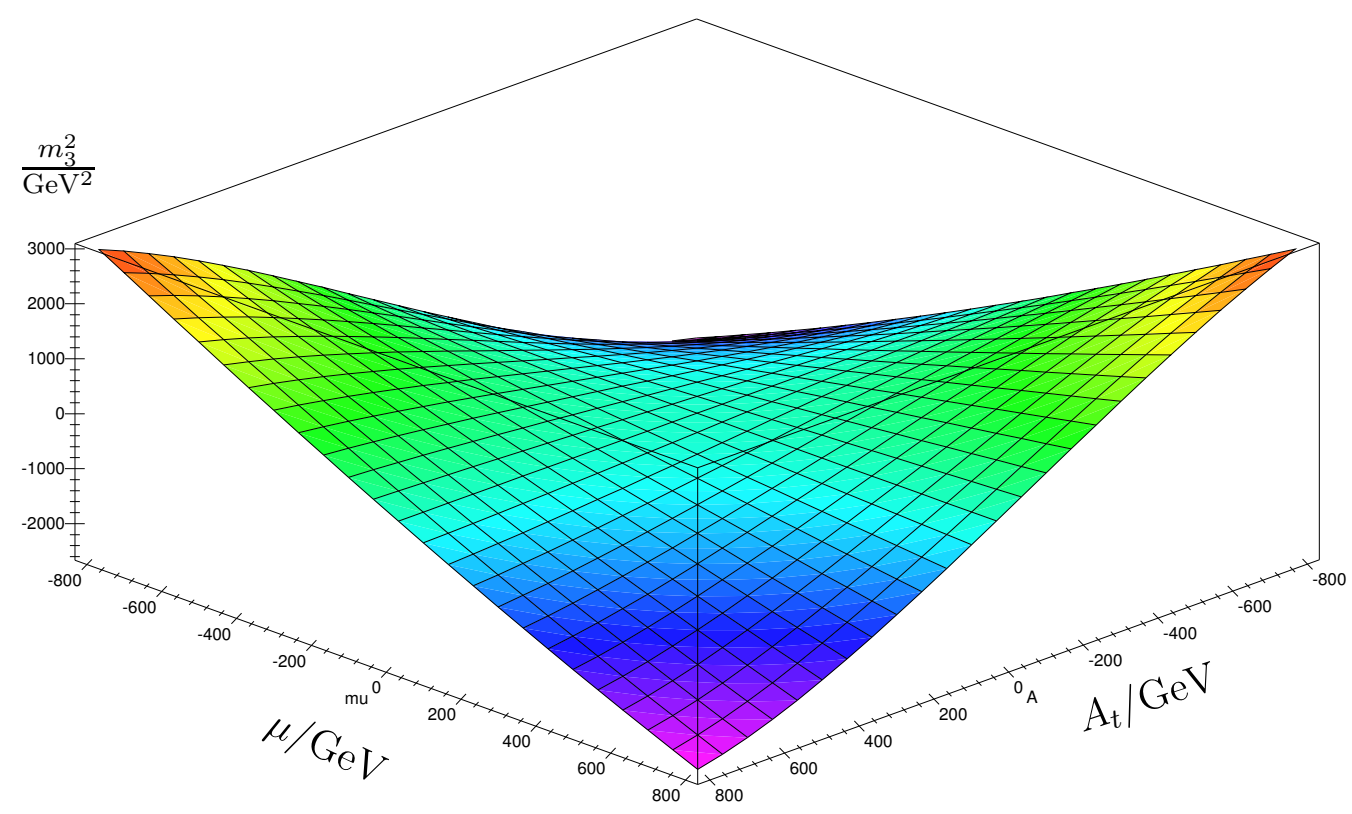

Figure 3: Dominant 1-loop effect: $m_{3}$ in dependence of $A_{t}$ and $\mu$ including stops and charginos. The tree level contribution causes a shift in the mean $m_{3}^{2}$. Large values with equal signs are preferred to give a negative contribution to $m_{3}^{2}$.

An interesting result we obtain by this method is that $m_{3}^{2}\left(h_{1}, h_{2}\right)$ tends to become larger while approaching the broken phase within the wall. The opposite behavior was noticed in [11]. An increasing $m_{3}^{2}$ is undesired since large phases in the broken minimum are restricted by the experiments. Also small phases are excluded by experimental bounds on the Higgs masses which are connected with small $\mathrm{CP}$ violation [8]. So even if a transitional $\mathrm{CP}$ phase occurs analytically it might be excluded by experiments.

The other parameters give minor effects which nevertheless can contribute. Large $M_{2}$ and $\mu$ are favored in order to get small $m_{3}^{2}$. As already mentioned, in this kind of analysis we always fix most of the parameter and vary only some of them. But we are interested in the whole parameter space. We will see that the shown dependences must be treated with caution since they may change using more general investigation methods. 
In the next stage we want to investigate the complete parameter space in a wide range. We therefore choose a different strategy. We will analyses correlations in average. At the first stage, we do not solve for $h_{1}, h_{2}, T_{c}$, but rather take them as free parameters in the ranges $h_{1} / T=-2 \ldots 2$ and $h_{2} / T=0 \ldots 2, T=80 \ldots 120$ $\mathrm{GeV}$. The zero temperature parameters are varied in the wide ranges

$$
\begin{aligned}
& \tan \beta=2 \ldots 20, \quad m_{A}=0 \ldots 400 \mathrm{GeV} \\
& m_{U}=-50 \ldots 800 \mathrm{GeV}, \quad m_{Q}=50 \ldots 800 \mathrm{GeV}, \\
& \mu, A_{t}, M_{1}, M_{2}=-800 \ldots 800 \mathrm{GeV} .
\end{aligned}
$$

Here a negative $m_{U}$ means in fact a negative right-handed stop mass parameter, $-\left|m_{U}^{2}\right|$. We have also studied separately the (dangerous [7]) region where the transition is very strong [3,5] corresponding to $m_{U} \sim-70 \ldots-50 \mathrm{GeV}$.

Note that since we do not solve for the equations of motion at this stage but allow for $h_{1}= \pm\left|h_{1}\right|$, we have to divide in (4.1) by $h_{1} h_{2}$ instead of $\left|h_{1} h_{2}\right|$ : this leads in general to positive values due to the tree-level form of the potential. A signal of a potentially promising region is then a small absolute value of the result, since this means that we are close to a point where $\partial_{\theta}^{2} V_{T}\left(h_{1}, h_{2}, \theta\right)$ crosses zero.

In Fig. 1 the relative number of $m_{3}^{2}$ versus its occurring values demonstrates which values are the most likely ones. There is a strong peak at a certain positive value and the probability decreases rapidly for zero or negative values of $m_{3}^{2}$. But nevertheless there are some negative $m_{3}^{2}$. It has to be checked, whether they are relevant for the bubble wall.

We found that spontaneous $\mathrm{CP}$ violation is statistically independent of the actual critical temperature. For checking the individual cases it may nevertheless play a role [12,13].

In the analytical result we observed that large $\mu$ and $A_{t}$ with equal signs are preferred. This can be checked in a two dimensional correlation plot in Fig. 6 . Both combinations are correlated with large positive $m_{3}^{2}$, hence with in average more stable regions. The best region is a cross-like region where either $A_{t}$ or $\mu$ are small. This could not be expected from the analytical approach which showed a preference of large values of $A_{t}$ and $\mu$.

The strongest correlations come from $A_{t}$ and $\mu$. The scale of their variation is larger by a factor of about 3 than the scales of the 1-loop mass parameter dependences with averaged 1-loop parameters.

Unfortunately small $m_{A}$ and large $\tan \beta$ are strongly preferred to obtain an 


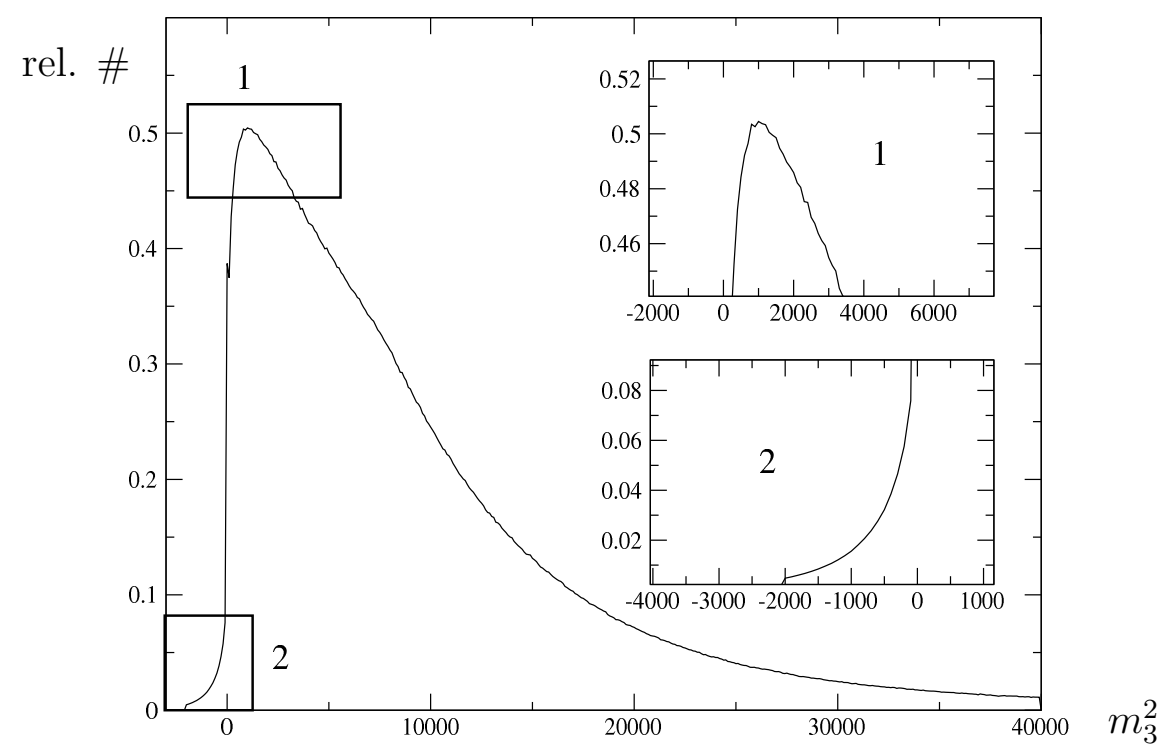

Figure 4: Relative number versus value of $m_{3}^{2}$. Small positive values are preferred.

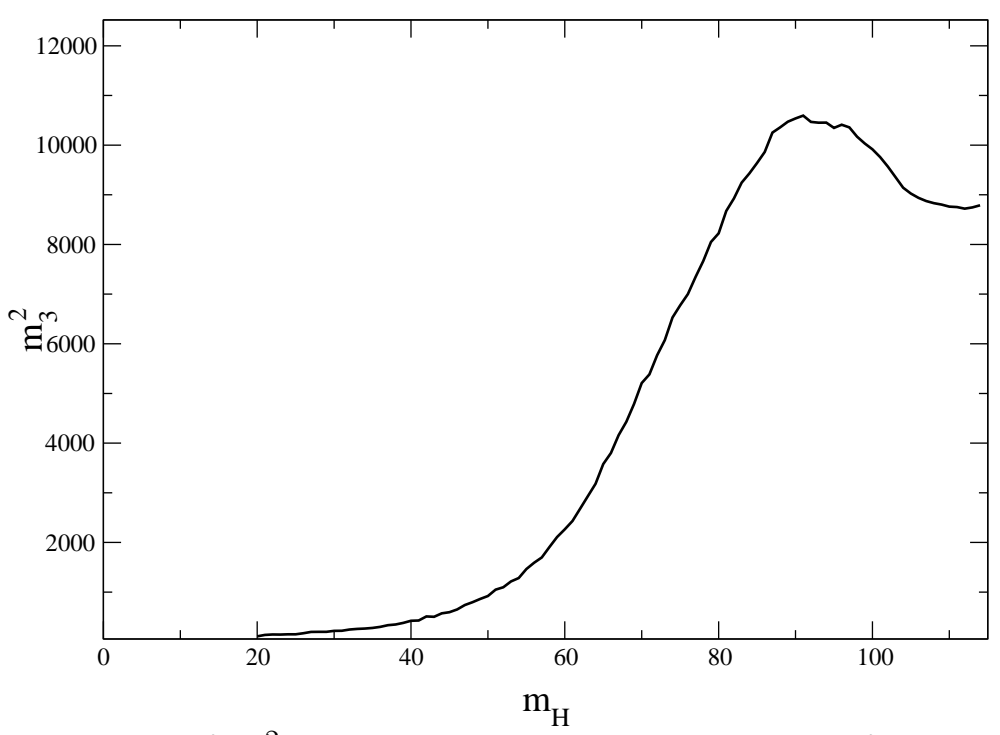

Figure 5: Correlation of $m_{3}^{2}$ and the Higgs mass $m_{H}$. Most favorable values are correlated with unphysical small Higgs values.

unstable $\mathrm{CP}$ direction. This is in contradiction to the requirements of a strong first order phase transition [4]. Moreover $\mathrm{CP}$ odd Higgs masses up to $m_{A} \sim$ $100 \mathrm{GeV}$ are experimentally excluded. 
In [8] it was argued that spontaneous $\mathrm{CP}$ violation at $T=0$ is excluded because of the experimental exclusion of the corresponding small Higgs masses. Accordingly, for transitional CP violation physical Higgs masses lower than around $40 \mathrm{GeV}$ are fairly probable, see Fig. 5. Here the average of $m_{3}^{2}$ decreases rapidly.

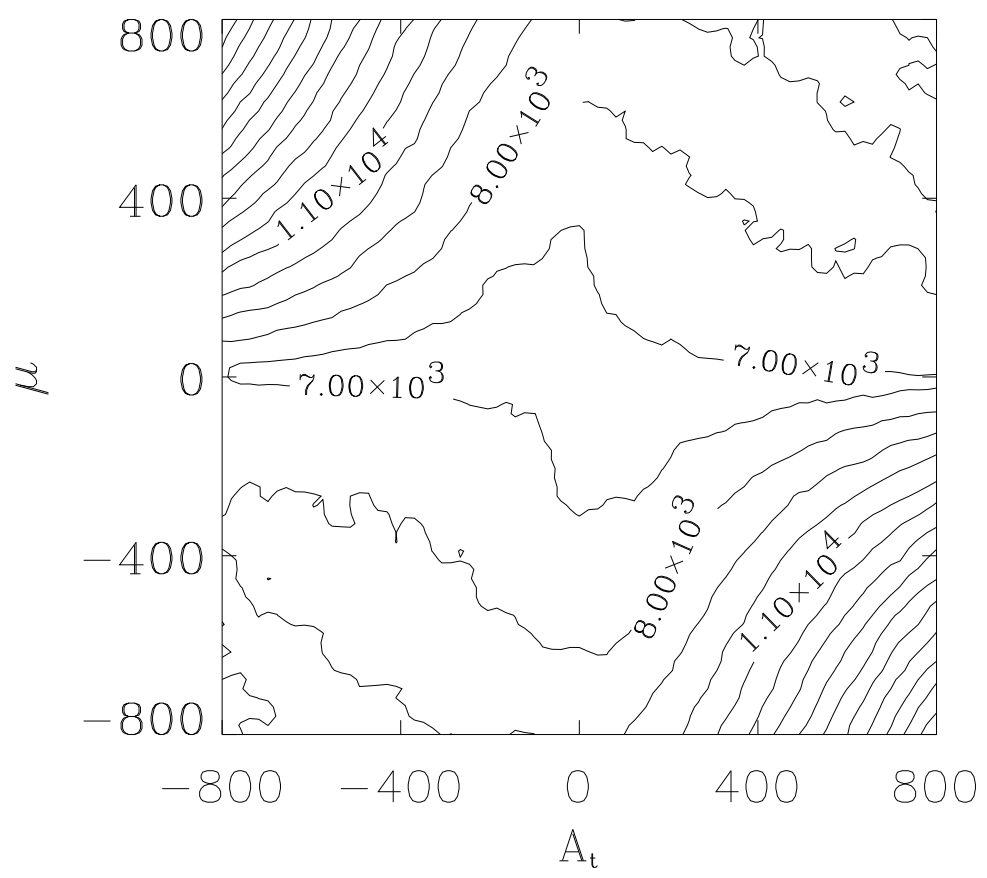

Figure 6: A totality of $1.03 \cdot 10^{9}$ averaged $m_{3}^{2}$ versus $A_{t}$ and $\mu$. The favorite region is the cross like region around vanishing $A_{t}$ and $\mu$.

There are also minor effects from the remaining parameters. The stop parameters $m_{U}$ and $m_{Q}$ are preferred to be small (Fig. 7a). The effect is smaller than the main 1-loop contribution from $A_{t}$ and $\mu . M_{1}$ and $M_{2}$ are almost completely uncorrelated. There are smaller correlations with $A_{t}$ or $\mu$ (Figs. 7b,c) which are folded into a vanishing correlation of $m_{3}^{2}$ with $M_{1}, M_{2}$ (Fig. 77d). The effects on $m_{U}, m_{Q}, M_{1}$, and $M_{2}$ do not strongly promote a small or negative $m_{3}^{2}$, but nevertheless they can be taken as hints.

In a second stage, we study the relevant parameter region in more detail. We start from the full parameter range (4.3) and restrict the search to the most favorable region. First, we determine the critical temperature. Then the equations of motion are solved for $\left(h_{1}, h_{2}\right)$. By comparing with the numerical exact results in several cases we find that a sufficient accuracy can be obtained by searching the "ridge" as an approximation, see Section 3. Finally, we look for the mini- 

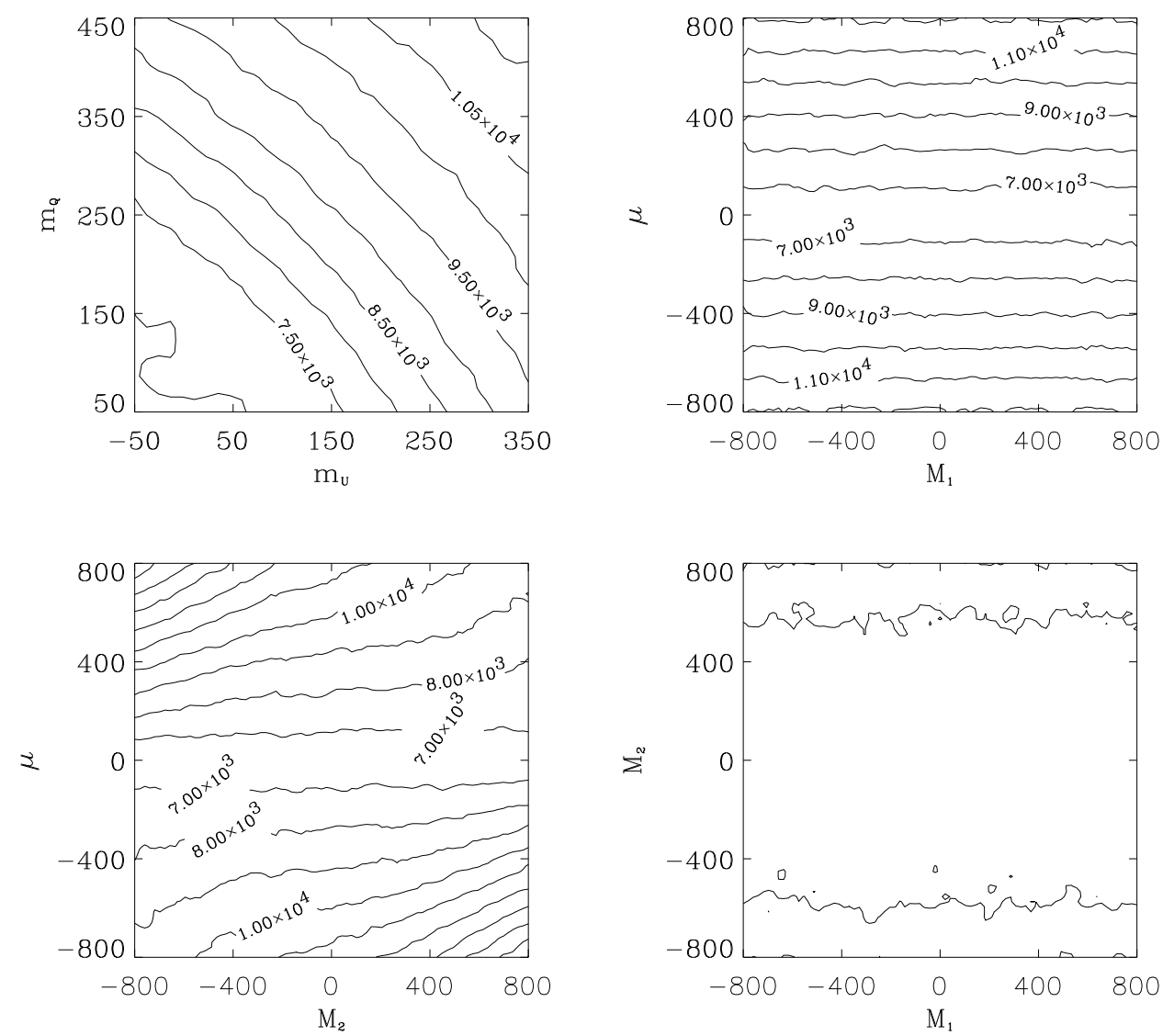

Figure 7: Correlations of the 1-loop parameters in average. The measure $m_{3}^{2}$ for instability versus the average of the other parameters is shown. The strongest correlation appears for the mass parameter $\mu . M_{1}$ and $M_{2}$ show less average dependences. Small $m_{U}$ are preferred, which coincides with the requirements of a strong phase transition whereas also small $m_{Q}$ are preferred, which points into the wrong direction.

mum in $\theta$ at fixed $\left(h_{1}, h_{2}\right)$ which is a fast and reliable approximation to the real solution as long as $\theta$ is small. We make some further restrictions: we exclude unphysical negative mass parameters as $m_{\tilde{t}}^{2}<0$ and discarded extremely weak phase transitions $v / T \ll 1$. We also excluded cases leading to $T=0$ spontaneous $\mathrm{CP}$ violation in the broken phase since this requires very small values of $m_{A}$ [8].

While in the statistical analysis we included $10^{9}$ parameter sets, we investigated $\sim 2 \times 10^{6}$ individual configurations in more detail. Nevertheless, we could not find any case with the desired property of temperature induced transitional CP violation within the corresponding bubble wall in the MSSM. Furthermore, in 
[11] a special point around $m_{U}^{2} \approx 0$ was considered. Since thermal mass were neglected, this corresponds to the point were $m_{U}^{2}+\# T^{2} \approx 0$. This point is in the vicinity of charge and color breaking minima. Without expanding the 1-loop contribution in $v_{1} / v_{2}$, we cannot reproduce the instable behavior proposed in [11].

Moreover we found that in the MSSM the dominating effects which support small $m_{3}^{2}$ are in contradiction to strong phase transitions and baryogenesis requirements.

\subsection{Explicit CP Violation}

We investigated also explicit CP violation in the wall. Although there are strong experimental constraints on the magnitude of the corresponding phases it might be interesting to see how strong the variation of $\theta$ along the phase boundary can be, and if it contributes considerably to the baryon asymmetry.

There are several possibilities to include explicit CP violation in the effective potential. In principle, several mass parameters might be complex. At tree level this is $m_{12}^{2}$, and at 1-loop level we have more possibilities through the masses of stop, chargino, and neutralino. In the stop, chargino, and neutralino sector $\mu, A_{t}$ and the soft breaking mass terms can carry phases:

$$
\begin{aligned}
\theta_{m_{12}} \leftrightarrow m_{12}^{2} & =\mu B, \quad \theta_{A_{t}} \leftrightarrow A_{t}, \quad \theta_{\mu} \leftrightarrow \mu, \\
\theta_{M_{1}} & \leftrightarrow M_{1}, \quad \theta_{M_{2}} \leftrightarrow M_{2} .
\end{aligned}
$$

Altogether we have five phases. $\theta_{m_{12}}$ can be absorbed in the Higgs fields and then arises in the mass matrices. The phases in the stop off diagonal entries can be absorbed into a complex $\tilde{A}_{t}=A_{t}-\mu \cot \beta$. We define the remaining three phases as $\theta_{A}=\theta_{m_{12}}+\theta_{A_{t}}+\theta_{\mu}, \theta_{C}=\theta_{m_{12}}+\theta_{\mu}+\theta_{M_{2}}$, and $\theta_{M}=\theta_{m_{12}}+\theta_{\mu}+\theta_{M_{1}}$ where $\theta_{A}$ appears in the stop matrix, $\theta_{C}$ in the chargino matrix, and together with $\theta_{M_{1}}$ in the neutralino matrix. In the chargino and stop eigenvalues we then have to replace $\cos \theta \rightarrow \cos \left(\theta+\theta_{A}\right)$ and $\cos \theta \rightarrow \cos \left(\theta+\theta_{C}\right)$. The replacement in the neutralino eigenvalues is more complicated and will not be given here.

Again we investigate the variation of $\theta$ along the wall. As in the spontaneous case, we search for minima along the $\theta$ direction. For baryogenesis, we need a strong variation of the phase $\partial_{x} \theta(x)$ along the wall. But even for maximal angles $\theta_{A}=\pi / 2, \theta_{C}=\pi / 2$, and $\theta_{M_{1}}=0$ we find a strongly suppressed CP phase in the broken Higgs phase. For $m_{A} \lesssim 90 \mathrm{GeV} \theta(x)$ is of order $10^{-3} \ldots 10^{-2}$ and varies only moderately within the wall as shown in Fig. 8. Especially there is no kind of peak as proposed earlier [11]. Only for experimentally excluded values of 
$m_{A} \lesssim 10 \mathrm{GeV}$ we do obtain phases up to order unity. For explicit phases of order $10^{-1}$ the dynamical phase is typically of order $10^{-4}$. We again realize that the size of the variation is dominated by the tree level parameters $m_{A}$ and $\tan \beta$.

From a technical point of view the evolution of the phase $\theta(x)$ into the symmetric phase is of interest. It is more difficult to determine (and less meaningful). The solutions of Fig. 8 have been obtained by using a tanh-ansatz also for $\theta(x)$, which turned out to compare very well with the numerical precision solution in the middle of the wall, where the full equations of motion can be solved more easily.

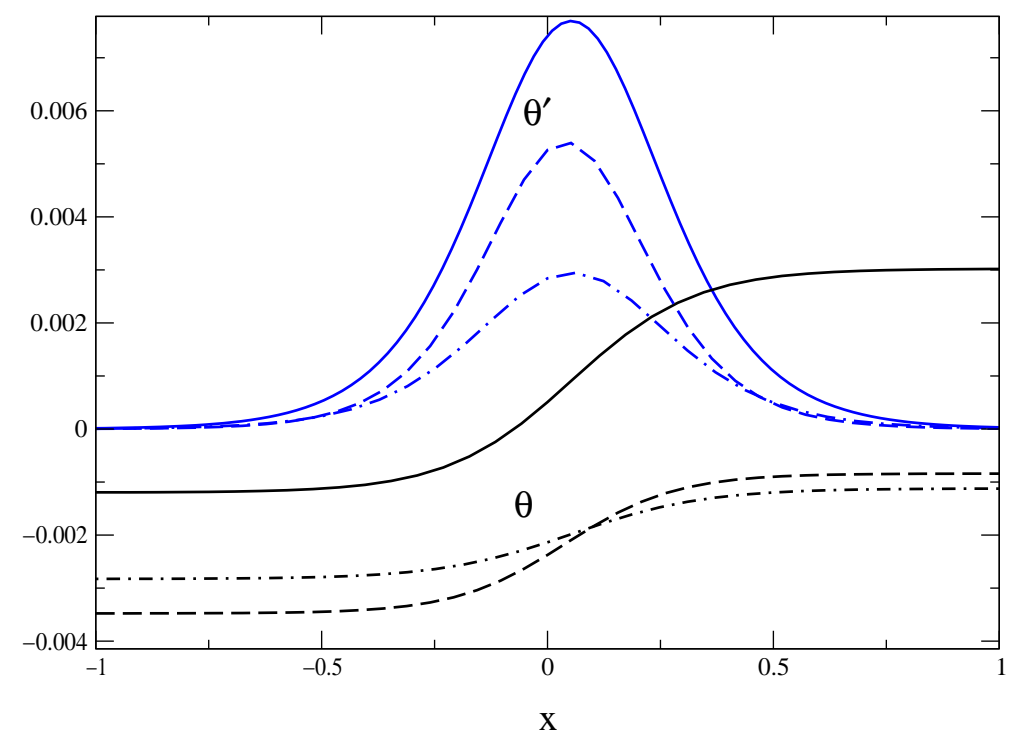

Figure 8: Dependence of $\theta$ and $\theta^{\prime}$ on $m_{A}$ near physically realistic cases: The derivative strongly depends on $m_{A}$ and $\tan \beta$. Solid: $m_{A}=80 \mathrm{GeV}, \tan \beta=2.0$, dashed: $m_{A}=120 \mathrm{GeV}, \tan \beta=2.0$, dot-dashed: $m_{A}=120 \mathrm{GeV}, \tan \beta=3.0$. $(x$ and $\theta^{\prime}$ in units of $G e V^{-1}$.)

\subsection{CP Violation in the NMSSM}

In the NMSSM we have many possibilities and a much larger parameter space [27]. Besides the Higgs fields $h_{1}$ and $h_{2}$ we have a singlet field $S$. Permitting complex fields, we can write the singlet field $S=n+i c$ implicitly introducing a phase $\theta_{s}=\arctan (c / n)$. Indeed, it turns out that transitional $\mathrm{CP}$ violation can occur in the NMSSM quite naturally. In [13] an example is shown where in the $S U(2) \times U(1)$-symmetric phase the singlet carries a vev in both components: 
$(n, c)_{\text {symm. }}=(50.4 G e V, 99 \mathrm{GeV})$ and $(n, c)_{\text {broken }}=(-122 \mathrm{GeV}, 0 \mathrm{GeV})$. Moreover the standard Higgs phase varies between zero and a value of order $\theta_{\text {sym }} \approx 1 / 20$ (in this special example) in the symmetric phase and zero in the broken phase. Both phases, $\theta$ and $\theta_{s}$ start with non-vanishing values in the symmetric phase and vanish in the broken phase.

We used the values $k=0.4, \lambda=0.05, \mu=212.6 \mathrm{GeV}, m_{Q}=278.1 \mathrm{GeV}$, $m_{U}=209.0 \mathrm{GeV}, B=83.9, r=533.2, \tan \beta=-5.0, A_{t}=-219.8 \mathrm{GeV}, A_{k}=$ $50 \mathrm{GeV}, A_{\lambda}=-150 \mathrm{GeV}, M_{1}=41.3 \mathrm{GeV}, M_{2}=82.2 \mathrm{GeV}$ [13, 28]. The critical temperature is $T_{c}=102.2 \mathrm{GeV}$.

This demonstrates the general possibility of transitional CP violation in bubble walls of the NMSSM phase transition. We have a strong phase transition in the example case. This shows the principle viability of baryogenesis in the NMSSM. More detailed calculations can be found in [28].

\section{The Baryon asymmetry in the MSSM in the semi-classical limit}

\subsection{Generalities}

As just discussed there can be explicit CP violation in the MSSM and also transitional CP violation in the NMSSM. The expanding bubble walls of a first order phase transition sweeping through the hot plasma generate temporary nonequilibrium. In the hot plasma we have sizeable baryon number violation by the weak sphaleron which rapidly stops in the equilibrium Higgs phase. Thus baryogenesis in principle is possible. However, we have to describe a concrete procedure since these are only necessary conditions. Technically one has to study transport equations in the plasma for particles/fields with varying mass due to the varying Higgs fields in the bubble wall. For thermal momenta $p \sim T$ and a "thick" wall $\left(L_{w} \gg 1 / T\right)$ the semi-classical approximation should be appropriate and we should end up with Boltzmann transport equations. In principle one has to start with the Baym-Kadanoff equations of the real time non-equilibrium formalism and end up with classical particle equations (plus some corrections as memory effects). In a different corner for soft bosonic fields a classical field description is expected. Here we only will deal with the particle limit and discuss the WKB approximation in accordance with refs. [16, 18]. Since we are mainly interested in the baryon asymmetry we will first calculate the transport for an 
asymmetry between left-handed particles and their CP conjugates and then consider baryogenesis via (out of equilibrium) sphaleron transitions in the symmetric phase. The transport equations for particles alone are interesting if the friction of the bubble wall and the resulting stationary wall velocity is calculated. Here $\mathrm{CP}$ violation can be neglected. As it will turn out that the wall velocity strongly influences baryogenesis this is an important point, not being dealt with here, but we refer to refs. 19, 24, 26.

\subsection{WKB approximation and dispersion relations}

In the following we summarize the derivation of the chargino dispersion relations in the presence of a slowly varying Higgs field background. For the details of the calculation we refer the reader to ref. [28] where also the application to squarks was worked out. Indeed the contribution of squarks turned out to be sub-leading.

To obtain the dispersion relation we solve the Dirac equation for the charginos in the WKB approximation

$$
\left(\begin{array}{cc}
-M & i \sigma^{\mu} \partial_{\mu} \\
i \bar{\sigma}^{\mu} \partial_{\mu} & -M^{\dagger}
\end{array}\right)\left(\begin{array}{l}
\Psi_{L} \\
\Psi_{R}
\end{array}\right)=0
$$

where

$$
M=\left(\begin{array}{cc}
M_{2} & g_{2}\left(H_{2}^{0}\right)^{*} \\
g_{2}\left(H_{1}^{0}\right)^{*} & \mu
\end{array}\right)
$$

and $\Psi_{L}=\left(\tilde{W}^{+}, \tilde{h}_{2}^{+}\right)^{T}, \Psi_{R}=\left(\overline{\tilde{W}^{-}}, \overline{\tilde{h}_{1}^{-}}\right)^{T}$. Since spontaneous CP violation does not occur in the MSSM, we have to rely on explicit CP violation by the $M_{2}$ and $\mu$ parameters. At this level all interactions between the particle and the plasma are neglected. The scattering effects will be accounted for in Section 5.3 when Boltzmann equations are written down to describe the local phase space distributions.

During the passage of the bubble wall the chargino mass matrix becomes spacetime dependent. We neglect the curvature of the wall and boost to the rest frame of the bubble wall. Then the mass matrix only depends on the position relative to the wall, i.e. $M=M(z)$.

Exploiting conservation of energy and boosting to the Lorentz frame where the momentum parallel to the wall vanishes, we take the ansatz $\Psi=e^{-i E t} \xi(z)$. Eq. (5.1) separates into two equations 17

$$
i \partial_{z} \xi_{ \pm}= \pm Q(z) \xi_{ \pm}, \quad Q(z)=\left(\begin{array}{cc}
E & -M^{\dagger}(z) \\
M(z) & -E
\end{array}\right)
$$


where $\xi_{ \pm}$are the $S_{z}= \pm \frac{1}{2}$ components of $\xi$. To solve Eq. (5.3) one brings the $z$-dependent matrix $Q(z)=D(z) Q_{D}(z) D(z)^{-1}$ into a diagonal form, where [28]

$$
\begin{aligned}
Q_{D} & =\left(\begin{array}{cc}
\sqrt{E^{2}-m^{2}} & 0 \\
0 & -\sqrt{E^{2}-m^{2}}
\end{array}\right), \\
D & =\left(\begin{array}{cc}
U & 0 \\
0 & V
\end{array}\right)\left(\begin{array}{cc}
\cosh X & e^{-i \theta} \sinh X \\
e^{i \theta} \sinh X & \cosh X
\end{array}\right) .
\end{aligned}
$$

The SU(2) matrices

$$
U=\left(\begin{array}{cc}
\cos a & e^{-i \gamma} \sin a \\
-e^{i \gamma} \sin a & \cos a
\end{array}\right), \quad V=\left(\begin{array}{cc}
\cos b & e^{-i \delta} \sin b \\
-e^{i \delta} \sin b & \cos b
\end{array}\right)
$$

are determined by $M=V M_{D} U^{\dagger}$, where $M_{D}=\operatorname{diag}\left(m_{1} e^{i \theta_{1}}, m_{2} e^{i \theta_{2}}\right)$. The entries of $X$ which is a diagonal $2 \times 2$ matrix in flavor space, obey tanh $2 X_{1,2}=m_{1,2} / E$. In the local WKB basis $\tilde{\xi}_{ \pm}=D^{-1} \xi_{ \pm}$the Dirac equation (5.3) takes the form

$$
i \hbar \partial_{z} \tilde{\xi}_{ \pm}=\left( \pm Q_{D}-D^{-1} i \hbar \partial_{z} D\right) \tilde{\xi}_{ \pm}
$$

which still is an exact equation. For mixing Dirac fermions like the charginos the transformation to the WKB basis consists of a rotation in flavor space (first factor in $D$ ) and a rotation to the helicity basis (second factor in $D$ ). In case of a single Dirac fermion, e.g. the top quark, there is only the helicity rotation [17,28], while for scalar particles, e.g. the stops, only the flavor rotation is present [28].

In general, the correction term $D^{-1} i \hbar \partial_{z} D$ caused by the position dependent field redefinition is not of diagonal form. The components of $\tilde{\xi}_{ \pm}$are still coupled. However, the off-diagonal part is proportional to $\partial_{z} D \sim D / L_{w}$. Typical momenta of the particles in the plasma are of the order of the temperature $T$, which is much larger than $1 / L_{w}$ for the bubbles under consideration. We therefore expand Eq. (5.6) in powers of $\partial_{z}$ or more precisely in powers of $\hbar$ (WKB approximation) that we already reintroduced for that reason.

To order $(\hbar)^{0}$ we can neglect the $D^{-1} \hbar i \partial_{z} D$ contribution. Thus the components of $\tilde{\xi}_{ \pm}$decouple in Eq. (5.6). Inserting the WKB ansatz for the fermion field

$$
\tilde{\xi}_{ \pm} \sim e^{-\frac{i}{\hbar} \int^{z} p_{z}\left(z^{\prime}\right) d z^{\prime}}
$$

into (5.6), we obtain the dispersion relations

$$
p_{z}(E)=\operatorname{sgn}\left(p_{z}\right) \sqrt{E^{2}-m^{2}} .
$$


Obviously, in the classical limit the $\theta$-dependence completely disappears, demonstrating that $\mathrm{CP}$ violation is indeed a quantum-mechanical phenomenon.

To solve the Dirac equation (5.6) to order $\hbar$ we have to take into account the $D^{-1} \hbar i \partial_{z} D$ term which reintroduces a coupling between the two components of $\tilde{\xi}_{ \pm}$. The dispersion relations $p_{z}(E)$ are obtained from the eigenvalues of the matrix $\pm Q_{D}-D^{-1} i \hbar \partial_{z} D$. Since to order $\hbar$ the off-diagonal terms do not contribute we are left with 16, 28,

$$
\begin{aligned}
& L_{I}: p_{z}=\operatorname{sgn}\left(p_{z}\right) \sqrt{E^{2}-m_{I}^{2}}-\left(\theta_{I}^{\prime}+\delta^{\prime} \sin ^{2} b\right) \sinh ^{2} X_{I}+\gamma^{\prime} \sin ^{2} a \cosh ^{2} X_{I}, \\
& \bar{L}_{I}: p_{z}=\operatorname{sgn}\left(p_{z}\right) \sqrt{E^{2}-m_{I}^{2}}+\left(\theta_{I}^{\prime}+\delta^{\prime} \sin ^{2} b\right) \sinh ^{2} X_{I}-\gamma^{\prime} \sin ^{2} a \cosh ^{2} X_{I}, \\
& R_{I}: p_{z}=\operatorname{sgn}\left(p_{z}\right) \sqrt{E^{2}-m_{I}^{2}}+\left(\theta_{I}^{\prime}-\gamma^{\prime} \sin ^{2} a\right) \sinh ^{2} X_{I}+\delta^{\prime} \sin ^{2} b \cosh ^{2} X_{I}, \\
& \bar{R}_{I}: p_{z}=\operatorname{sgn}\left(p_{z}\right) \sqrt{E^{2}-m_{I}^{2}}-\left(\theta_{I}^{\prime}-\gamma^{\prime} \sin ^{2} a\right) \sinh ^{2} X_{I}-\delta^{\prime} \sin ^{2} b \cosh ^{2} X_{I},
\end{aligned}
$$

where $I=1,2$ and $\theta_{I}^{\prime}=\partial_{z} \theta_{I}$, etc. and

$$
\sinh ^{2} X_{I}=\left(E-\sqrt{E^{2}-m^{2}}\right) / 2 \sqrt{E^{2}-m^{2}} .
$$

In the symmetric phase $L_{2}$ and $\bar{R}_{2}$ evolve to the left-handed Higgsinos states $\tilde{h}_{2}^{+}$and $\tilde{h}_{1}^{-}$, respectively. The flavor transformations $U$ and $V$ are related to the parameters of the chargino mass matrix (5.2):

$$
\begin{aligned}
& \sin ^{2} a=2|A|^{2} / \Lambda(\Lambda+\Delta) \quad \text { with } \\
& A=g_{2}\left(\left(M_{2} H_{2}^{0}\right)^{*}+\mu H_{1}^{0}\right) \\
& \Delta=\left|M_{2}\right|^{2}-|\mu|^{2}+g_{2}^{2}\left(\left|H_{1}^{0}\right|^{2}-\left|H_{2}^{0}\right|^{2}\right) \\
& \Lambda=\left(\Delta^{2}+4|A|^{2}\right)^{1 / 2}
\end{aligned}
$$

and $\gamma=\arg (\mathrm{A})$. This gives

$$
\gamma^{\prime} \sin ^{2} a=2 \operatorname{Im}\left(A^{*} A^{\prime}\right) / \Lambda(\Lambda+\Delta)
$$

and there are similar relations for $\sin ^{2} b, \delta$ and $\delta^{\prime} \sin ^{2} b$ exchanging $a$ and $b, H_{1}^{0}$ and $H_{2}^{0}, \gamma$ and $-\delta$. The mass eigenvalues (in non-symmetric notation) read

$$
\begin{aligned}
& {\left[M_{D}\right]_{11}=M_{2} \frac{\cos a}{\cos b}-g_{2}\left(H_{2}^{0}\right)^{*} \frac{\sin a}{\cos b} e^{-i \gamma},} \\
& {\left[M_{D}\right]_{22}=\mu \frac{\cos a}{\cos b}+g_{2}\left(H_{1}^{0}\right)^{*} \frac{\sin a}{\cos b} e^{i \gamma} .}
\end{aligned}
$$

Notice that the variation of $m_{I}$, which is encoded in $\partial_{z} X_{I}, \partial_{z} a$ and $\partial_{z} b$ drops in the dispersion relations. The $\mathrm{CP}$ violating part of the dispersion relation is 
proportional to the derivative of the phases $\theta_{I}, \gamma$ and $\delta$. Thus only varying phases contribute to $\mathrm{CP}$ violation in the semi-classical limit. Furthermore, CP violation is proportional to derivatives which guarantees that its effect is turned off far away from the bubble wall. Because of the different dispersion relations, left- and righthanded particles feel a different (semi-classical) force in their interaction with the wall. For the anti-particles, $\bar{L}$ and $\bar{R}$, the CP violating part comes with the opposite sign.

As pointed out very recently in ref. [18] one should use the kinetic momentum $p_{\text {kin }}=m V_{\text {group }}=m \frac{\partial E}{\partial p}$ instead of the canonical momentum $p$ (we used up to now) in the classical limit leading to Boltzmann equations, quite in the spirit of the correspondence principle of basic quantum mechanics. Calculating the (inverse of the) group velocity from (5.9) $E$-independent terms drop out, i.e. $\cosh ^{2} X_{I}$ can be substituted by $\sinh ^{2} X_{I}$. The kinetic momenta beyond the zeroth order (5.8) then contain correction terms $\pm\left(\theta_{I}^{\prime}+\delta^{\prime} \sin ^{2} b-\gamma^{\prime} \sin ^{2} a\right) m^{2} /\left(2 E\left(E^{2}-m^{2}\right)^{1 / 2}\right)$. Thus indeed kinetic and canonical momentum are not equal because of $\mathrm{CP}$ violating effects.

The dispersion relations can be inverted. To leading order in the derivatives the CP violating part of the dispersion relation for the eigenstate $L_{2}$ which in the symmetric phase corresponds to $\tilde{h}_{2}^{+}$is

$$
\Delta E=-\operatorname{sgn}\left(p_{z}\right)\left(\theta_{2}^{\prime}+\delta^{\prime} \sin ^{2} b-\gamma^{\prime} \sin ^{2} a\right) m^{2} / 2\left(p_{\text {kin }}^{2}+m^{2}\right) .
$$

(For $p_{\text {kin }} \gg m$ this is twice the result one would obtain with the canonical momentum after substituting $\cosh ^{2}$ by $\sinh ^{2}$ in (5.9).) Most importantly $\Delta E$ is now totally symmetric under the exchange of $H_{1}$ and $H_{2}$. This will destroy the most prominent source term $\sim H_{1} H_{2}^{\prime}-H_{1}^{\prime} H_{2}$ of older work.

The phases $\gamma$ and $\delta$ only vary due to a change in the Higgs vev ratio $\tan \beta$ or because of transitional $\mathrm{CP}$ violation in the bubble wall. The first contribution is suppressed, since the variation of $\beta$ is at most $\sim 10^{-2}$ for realistic Higgs masses [34, 38], while transitional CP violation most probably does not occur at all in the MSSM as discussed in Section 4.1 [13]. On the other hand, the contribution to the chargino dispersion relations stemming from the variation of the complex phases in $\mathbf{M}_{D}$ only requires explicit CP violating phases in $\mu$ or $M_{2}$. Eq. (5.12) demonstrates that even though the phases in the two terms entering $\left[M_{D}\right]_{11,22}$ are position independent, their contribution to the resulting phase varies due to the change in the (real) Higgs vevs. 


\subsection{Diffusion equations and the baryon asymmetry}

In this Section we study the coupled differential equations that describe particle interactions and transport during the phase transition. We treat the plasma as consisting of quasi-classical particles with definite canonical position and momentum. The phase space distributions $f_{i}(\vec{x}, \vec{p}, t)$ of the particles evolve according to the classical Boltzmann equation

$$
d_{t} f_{i}=\left(\partial_{t}+\dot{\vec{x}} \cdot \partial_{\vec{x}}+\dot{\vec{p}} \cdot \partial_{\vec{p}}\right) f_{i}=\mathcal{C}_{i}[f]
$$

The time derivatives of position and momentum obey the Hamilton equations $\dot{\vec{x}}=\partial_{\vec{p}} E(\vec{x}, \vec{p})$ and $\dot{\vec{p}}=-\partial_{\vec{x}} E(\vec{x}, \vec{p})$, where $E(\vec{x}, \vec{p})$ are the dispersion relations derived in the previous section. This treatment is an approximation to quantum Boltzmann equations which have to be discussed in principle. This picture is justified for thick walls $\left(p \gg 1 / L_{w}\right)$ if it predicts a sizable effect, not dominated by non-leading terms in the derivative expansion [20].

The Boltzmann equation can in principle be solved numerically. However, to make it analytically tractable we use the fluid-type truncation [17

$$
f_{i}(\vec{x}, \vec{p}, t)=\frac{1}{e^{\beta\left(E_{i}-v_{i} p_{z}-\mu_{i}\right)} \pm 1}
$$

for the phase space densities of fermions $(+)$ and bosons $(-)$ in the rest frame of the plasma. Here $v_{i}$ and $\mu_{i}$ denote the velocity perturbations and chemical potentials for each fluid, respectively. We also split $E_{i}$ into a dominant part $E_{0 i}=\sqrt{p^{2}+m_{i}^{2}}$ and a perturbation $\Delta E_{i} \sim \partial_{z} \theta$ which is related to CP violation. The chemical potentials are the central quantities that finally will determine the baryon asymmetry. The velocity perturbation on the other hand, is only introduced to allow the particles to move in response to the force, giving rise to chemical potential perturbations.

We are looking for a "stationary" solution of the Boltzmann equation, because at late times the wall moves with constant velocity $v_{w}$. This means that any explicit time dependence enters in the combination $\bar{z} \equiv z-v_{w} t$. Inserting the fluid ansatz into the Boltzmann equation (5.14), eliminating $v_{i}$ and taking the difference between particles and anti-particles, we obtain to linear order in the perturbations $\Delta E_{i}$ and $\mu_{i}$, and to leading order in the wall velocity [16]

$$
\begin{array}{r}
-\kappa_{i}\left(D_{i} \mu_{i}^{\prime \prime}+v_{w} \mu_{i}^{\prime}\right)+\sum_{p} \Gamma_{p}^{d} \sum_{j} \mu_{j}=S_{i}, \\
S_{i}=\frac{D_{i} v_{w}}{\left\langle p_{z}^{2} / E_{0}\right\rangle_{0}}\left\langle p_{z} \Delta E_{i}^{\prime}\right\rangle^{\prime}
\end{array}
$$


where primes denote $\partial_{\bar{z}}$. The diffusion constants read $D_{i}=\kappa_{i}\left\langle p_{z}^{2} / E_{0}\right\rangle_{0}^{2} /\left(\bar{p}_{z}^{2} \Gamma_{i}^{e}\right)$, and $\Gamma^{e}, \Gamma^{d}$ are the rates for elastic and inelastic processes, respectively. The averages are carried out according to

$$
\langle\cdot\rangle \equiv \frac{\int d^{3} p f_{ \pm}^{\prime}(\cdot)}{\int d^{3} p f_{+}^{\prime}(m=0)} \equiv \kappa_{i} \frac{\int d^{3} p f_{ \pm}^{\prime}(\cdot)}{\int d^{3} p f_{ \pm}^{\prime}} .
$$

Here $f_{ \pm}^{\prime}=d f_{ \pm} / d E_{0}=-\beta e^{\beta E_{0}} /\left(e^{\beta E_{0}} \pm 1\right)^{2}$ is the derivative of the unperturbed Fermi-Dirac or Bose-Einstein distribution. The statistical factor $\kappa$ is 1 for massless fermions, 2 for massless bosons and exponentially small for particles much heavier than $T$. The subscript " 0 " denotes averaging with the massless, unperturbed Fermi-Dirac distribution.

The CP violating source term $S_{i}$ in (5.16), which is due to the semi-classical force, is proportional to the diffusion constant. This is because particles must move in order to build up perturbations. In $S_{i}$ the thermal averages over the CP violating energy perturbations $\Delta E$ are performed using the massive distribution functions in order to account for Boltzmann suppression of heavy particles.

We obtain the transport equations of the MSSM from Eq. (5.16) by specifying the relevant particle species and interactions in the hot plasma. The network of equations can be simplified considerably by using conservation laws and neglecting interactions that are slow compared to the relevant time scale, which requires $D / v_{w}^{2} \ll \Gamma^{-1}$.

In a first step we neglect the weak sphaleron interaction with rate $\Gamma_{w s}$, which will be included at the end of the calculation. In the following we will therefore assume baryon and lepton number conservation. The neglect of the weak sphalerons allows us to completely forget about leptons in our transport equations and compute only the quark and Higgs densities.

We assume the supergauge interactions to be in equilibrium. The chemical potential of any particle is then equal to that of its superpartner, and it is convenient to define the chemical potentials $\mu_{U}=\left(\mu_{u^{c}}+\mu_{\tilde{u}^{c}}\right) / 2, \mu_{Q_{1}}=\left(\mu_{u}+\mu_{d}+\mu_{\tilde{u}}+\mu_{\tilde{d}}\right) / 4$, $\mu_{H_{1}}=\left(\mu_{H_{1}^{0}}+\mu_{H_{1}^{-}}+\mu_{\tilde{h}_{1}^{0}}+\mu_{\tilde{h}_{1}^{-}}\right) / 4$, etc. Furthermore, we take into account the following interactions

$$
\begin{aligned}
& \left(\Gamma_{y}+\Gamma_{y A}\right)\left(\mu_{H_{2}}+\mu_{Q_{3}}+\mu_{T}\right), \quad \Gamma_{y \mu}\left(\mu_{H_{1}}-\mu_{Q_{3}}-\mu_{T}\right), \\
& \Gamma_{s s}\left(2 \mu_{Q_{3}}+2 \mu_{Q_{2}}+2 \mu_{Q_{1}}+\mu_{T}+\mu_{B}+\mu_{C}+\mu_{S}+\mu_{U}+\mu_{D}\right), \\
& \Gamma_{h f}\left(\mu_{H_{1}}+\mu_{H_{2}}\right), \quad \Gamma_{m}\left(\mu_{Q_{3}}+\mu_{T}\right), \quad \Gamma_{H_{1}} \mu_{H_{1}}, \quad \Gamma_{H_{2}} \mu_{H_{2}} .
\end{aligned}
$$

The rates in the first line are related to the Lagrangian

$$
\mathcal{L}_{\text {int }}=y_{t} t^{c} q_{3} H_{2}+y_{t} \tilde{t}^{c} q_{3} \tilde{h}_{2}+y_{t} t^{c} \tilde{q}_{3} \tilde{h}_{2}-y_{t} \mu \tilde{t}^{c *} \tilde{q}_{3}^{*} H_{1}+y_{t} A_{t} \tilde{t}^{c} \tilde{q}_{3} H_{2}+\text { h.c. }
$$


$\Gamma_{s s}$ denotes the strong sphaleron rate. $\Gamma_{h f}$ is due to Higgsino helicity flips induced by the $\mu \tilde{h}_{1} \tilde{h}_{2}$ term. $\Gamma_{H_{1,2}}$ and $\Gamma_{m}$ correspond to Higgs and axial top number violating processes, present only in the phase boundary and the broken phase.

If the system is near thermal equilibrium, number densities and chemical potentials are related by

$$
n_{i}=\frac{1}{6} k_{i} \mu_{i} T^{2}
$$

where $k_{i}$ is the appropriate sum over statistical factors $\kappa$ introduced in (5.17), e.g. $k_{Q_{1}}=N_{c}\left(\kappa_{u}+\kappa_{d}+\kappa_{\tilde{u}}+\kappa_{\tilde{d}}\right), k_{U}=N_{c}\left(\kappa_{u^{c}}+\kappa_{\tilde{u}^{c}}\right), k_{H_{1}}=\left(\kappa_{H_{1}^{0}}+\kappa_{H_{1}^{-}}+\kappa_{\tilde{h}_{1}^{0}}+\kappa_{\tilde{h}_{1}^{-}}\right)$, etc. $N_{c}=3$ denotes the number of colors. In the massless limit one obtains $k_{Q_{1,2,3}}=18, k_{U}=k_{D}=\ldots=k_{T}=9, k_{H_{1,2}}=6$.

Using baryon number conservation and neglecting the small Yukawa couplings of the first and second family quarks the strong sphaleron rate reads

$$
\Gamma_{s s}\left(2 \mu_{Q_{3}}+\cdots+\mu_{D}\right)=\Gamma_{s s}\left[\left(2+9 \frac{k_{Q_{3}}}{k_{B}}\right) \mu_{Q_{3}}+\left(1-9 \frac{k_{T}}{k_{B}}\right) \mu_{T}\right]
$$

To arrive at this expression we made the assumption that all the squark partners of the light quarks are degenerate in mass. Assuming equilibrium for the strong sphalerons we obtain

$$
\mu_{T}=\frac{2 k_{B}+9 k_{Q_{3}}}{9 k_{T}-k_{B}} \mu_{Q_{3}} .
$$

The reduced set of diffusion equations for the relevant particle species then finally reads 28

$$
\begin{aligned}
-A \mathcal{D}_{q} \mu_{Q_{3}}+\left(\Gamma_{y}+\Gamma_{y A}\right)\left[\mu_{H_{2}}+B \mu_{Q_{3}}\right]-\Gamma_{y \mu}\left[\mu_{H_{1}}-B \mu_{Q_{3}}\right]+B \Gamma_{m} \mu_{Q_{3}} & =0 \\
-k_{H_{1}} \mathcal{D}_{h} \mu_{H_{1}}+\Gamma_{y \mu}\left[\mu_{H_{1}}-B \mu_{Q_{3}}\right]+\Gamma_{h f}\left(\mu_{H_{1}}+\mu_{H_{2}}\right)+\Gamma_{H_{1}} \mu_{H_{1}} & =S_{H_{1}} \\
-k_{H_{2}} \mathcal{D}_{h} \mu_{H_{2}}+\left(\Gamma_{y}+\Gamma_{y A}\right)\left[\mu_{H_{2}}+B \mu_{Q_{3}}\right]+\Gamma_{h f}\left(\mu_{H_{1}}+\mu_{H_{2}}\right)+\Gamma_{H_{2}} \mu_{H_{2}} & =S_{H_{2}}(5.2
\end{aligned}
$$

where

$$
\begin{aligned}
A & =\frac{9 k_{T} k_{Q_{3}}+9 k_{B} k_{Q_{3}}+4 k_{B} k_{T}}{9 k_{T}-k_{B}} \\
B & =\frac{k_{B}+9 k_{T}+9 k_{Q_{3}}}{9 k_{T}-k_{B}}
\end{aligned}
$$

and $\mathcal{D}_{i} \equiv D_{i} \frac{d^{2}}{d \bar{z}^{2}}+v_{w} \frac{d}{d \bar{z}}$.

We keep the rates related to the top Yukawa interactions finite. If these interaction are in equilibrium, the resulting diffusion equations are sourced only 
by the combination $S_{H_{1}}-S_{H_{2}}$, because of the constraint $\mu_{H_{1}}+\mu_{H_{2}}=0$. As a result the dominant contribution to the chargino source terms stemming from the $\theta$-dependent part in the dispersion relations (5.9) cancels, because the corresponding terms for $\tilde{h}_{1}^{-}$and $\tilde{h}_{2}^{+}$are exactly of the same size. This is not true for the $\gamma^{\prime}, \delta^{\prime}$ contributions which arise from the "flavor" transformations $\mathbf{U}$ and V. However, they are suppressed by the small variation of $\tan \beta$ in the bubble wall [6, 34, 38.

The full diffusion equations (5.23) have already been studied in ref. [18], with source terms corresponding to the helicity part of the dispersion relations (5.9). In the following we also include the flavor part of the source term in the analysis.

Before solving the network of diffusion equations, we turn to baryon number generation by the weak sphaleron processes. The evolution of the baryon number density $n_{\mathcal{B}}$ is governed by

$$
-\mathcal{D}_{q} n_{\mathcal{B}}+3 \Theta(\bar{z}) \Gamma_{w s}\left(T^{2} \mu_{\mathcal{B}_{L}}-a n_{\mathcal{B}}\right)=0
$$

where we have assumed identical diffusion constants for all quarks and squarks, and neglected contributions of leptons. The position dependence of the weak sphaleron rate is modeled by a step function $\Theta(\bar{z})$ : anomalous baryon number violation is unsuppressed in the symmetric phase $(\bar{z}>0)$ and suddenly switched off in the broken phase $(\bar{z}<0)$. Baryon number generation is sourced by the chemical potential of left-handed quarks

$$
\mu_{\mathcal{B}_{L}}=C \mu_{Q_{3}} \equiv\left[1-\frac{k_{Q_{3}}+2 k_{T}}{9 k_{T}-k_{B}}\left(\frac{2 k_{B}}{k_{Q_{1}}}+\frac{2 k_{B}}{k_{Q_{2}}}\right)\right] \mu_{Q_{3}} .
$$

The second term in Eq. (5.25) describes damping of the baryon asymmetry by weak sphalerons in the symmetric phase. The parameter $a$ depends on the degrees of freedom present in the hot plasma. Taking only the right-handed stop to be light gives $a=48 / 7$ [18].

From Eq. (5.25) one can easily obtain the baryon to entropy ratio in the broken phase

$$
\eta_{B} \equiv \frac{n_{\mathcal{B}}}{s}=\frac{135 \Gamma_{w s}}{2 \pi^{2} g_{*} v_{w} T} \int_{0}^{\infty} d \bar{z} \mu_{\mathcal{B}_{L}}(\bar{z}) e^{-\nu \bar{z}}
$$

where we have taken the entropy density $s=\left(2 \pi^{2} g_{*} / 45\right) T^{3}$ and $\nu=3 a \Gamma_{w s} /\left(2 v_{w}\right)$ [18]. $g_{*} \sim 126$ is the effective number of degrees of freedom at the phase transition temperature. Eq. (5.27) shows that the integral over the left-handed quark 
number, $n_{L} \propto \mu_{\mathcal{B}_{L}}$ in the symmetric phase determines the final baryon asymmetry.

We now return to eqs. (5.23) in order to compute $\mu_{\mathcal{B}_{L}}$. These linear second order differential equations can be solved by finding the appropriate Greens function. In contrast to ref. [18] we do not make any approximations in computing this Greens function. We keep the discussion general and consider the following set of $N$ coupled diffusion equations

$$
\left(\begin{array}{ccc}
-k_{11} \mathcal{D}_{11}+\Gamma_{11} & \cdots & -k_{1 N} \mathcal{D}_{1 N}+\Gamma_{1 N} \\
\vdots & \ddots & \vdots \\
-k_{N 1} \mathcal{D}_{N 1}+\Gamma_{N 1} & \cdots & -k_{N N} \mathcal{D}_{N N}+\Gamma_{N N}
\end{array}\right)\left(\begin{array}{c}
\mu_{1} \\
\vdots \\
\mu_{N}
\end{array}\right)=\left(\begin{array}{c}
S_{1} \\
\vdots \\
S_{N}
\end{array}\right)
$$

where $\mathcal{D}_{a b}=D_{a b} \frac{d^{2}}{d \bar{z}^{2}}+v_{w} \frac{d}{d \bar{z}}$. The corresponding boundary conditions read $\mu_{a}(|\bar{z}| \rightarrow$ $\infty)=0$. The matrix valued Greens function $G_{a b}$ is defined by $\sum_{c=1}^{N}\left(-k_{a c} \mathcal{D}_{a c}+\right.$ $\left.\Gamma_{a c}\right) G_{c b}(\bar{z})=\delta_{a b} \delta(\bar{z})$. In the transport equations (5.23) position dependent rates are present, e.g. $\Gamma_{m}$. They typically vanish in the symmetric phase and become maximal in the broken phase. In order to keep the problem analytically tractable we simply model the position dependence of these rates by step functions, i.e. $\Gamma_{a b}(\bar{z})=\Gamma_{+a b} \Theta(\bar{z})+\Gamma_{-a b} \Theta(-\bar{z})$.

\section{Numerical results}

In this section we present our numerical results for the baryon asymmetry in the MSSM, which has to be compared with the observational value $2-7 \times 10^{-11}$ [54]. We model the Higgs field $H_{1}(z)$ in the bubble wall by a kink with width $L_{w}$ and take $H_{2}(z)=\tan \beta(z) H_{1}(z)$, where $\tan \beta$ varies in the wall. In all our evaluations we assume $v_{c}=120 \mathrm{GeV}$ and $\tan \beta\left(T_{c}\right)=3$. For the critical temperature we take $T_{c}=90 \mathrm{GeV}$.

In Section 5.3 we emphasized the impact of the squark spectrum on the baryon asymmetry. A strong phase transition requires the right-handed stop to be light. In the transport equations we simply treat it as a massless particle, i.e. $k_{T}=18$. We assume all other sfermions to be heavy compared to $T_{c}$, i.e. $k_{Q_{3}}=k_{B}=$ 9. From Eq. (5.24) we find for the effective statistical factors in the transport equation $A=126 / 13$ and $B=23 / 13$, and from Eq. (5.26) $C=5 / 13$. This non-universal squark spectrum has the additional virtue of relaxing the strong sphaleron suppression of the baryon asymmetry [55]. The Higgs fields are treated as massless as well, i.e $k_{H}=6$. 

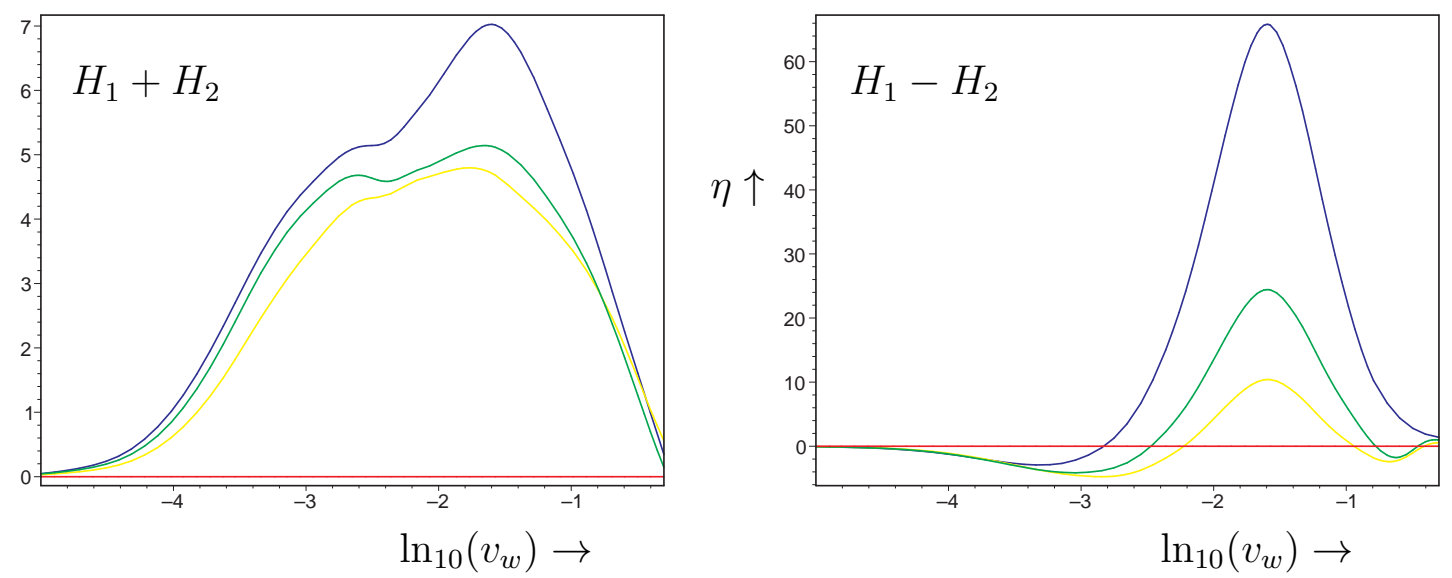

Figure 9: $\quad H_{1} \pm H 2$ contributions to the baryon asymmetry dependent on $v_{w}$ for different values of the wall thickness $L_{w}=20 / T, 15 / T, 10 / T$ (from below) and $|\mu|=\left|M_{2}\right|=150 \mathrm{GeV}, \arg \left(\mu M_{2}\right)=\pi / 2$ and $\delta \beta=0.01 . \eta$ is given in units of $2 \times 10^{-11}$ (observational bound).

For the diffusion constants of quarks and Higgses we take $D_{h}=110 / T$ and $D_{q}=6 / T$. The rates $\Gamma_{y A}$ and $\Gamma_{y \mu}$ which involve heavy squarks are Boltzmann suppressed and we set them to zero in the following [23]. Furthermore we take $\Gamma_{y}=0.015 T, \Gamma_{h f}=0.016 T, \Gamma_{m}=0.05 T \theta(-z), \Gamma_{H_{1}}=\Gamma_{H_{2}}=0.05 T \theta(-z)$. The weak sphaleron rate is $\Gamma_{w s}=20 \alpha_{w}^{5} T$.

In the following we separately present the $H_{1}-H_{2}$ and $H_{1}+H_{2}$ contributions to the baryon asymmetry, which originate from the flavor and helicity contributions to the chargino dispersion relation, respectively. We use the dispersion relations in the canonic momentum. In the formulation with the kinetic momentum the $H_{1}-H_{2}$ part vanishes [18]. However, a $H_{1}-H_{2}$ contribution has been found in real time Green's function treatments of the chargino current, most recently in ref. [23]. It would we very interesting to understand more clearly the relation between our $H_{1}-H_{2}$ source and the one found in that approach.

In fig. 9 we summarize our results for the baryon asymmetry generated during the phase transition. To maximize the result we take $|\mu|=\left|M_{2}\right|$ and maximal CP violation $\arg \left(M_{2} \mu\right)=\pi / 2$. For smaller phases the result simply scales with $\sin \left(\arg \left(M_{2} \mu\right)\right)$. Moreover, the $H_{1}-H_{2}$ result is proportional to the change in the Higgs vev ratio in the bubble wall, which we take to be $\delta \beta=0.01$. We work with $\mu=150$ and $\tan \beta=3$. The mass of the lightest chargino eigenstate is then about $110 \mathrm{GeV}$, in agreement with the experimental constraints.

In our evaluations we vary the wall velocity in the interval $10^{-5}<v_{w}<0.5$. For small values of $v_{w}$ we deal with a quasi equilibrium situation, and the baryon asymmetry goes to zero. Also large wall velocities suppress $\eta$, since transport 
in front of the bubble wall becomes inefficient. From Fig. 9 we observe that baryogenesis is most efficient if $v_{w}$ is a few times $10^{-2}$. This behavior was already found in refs. [18, 23]. Most interesting, calculations of the wall velocity in the MSSM lead to $v_{w}$ indeed in that range [19, 25]. The detailed calculations of ref. [25] lead tof $v_{w}=2-3 \cdot 10^{-2}$, very near to the maximum for $\eta$.

We also present results for different wall widths $L_{w}=10 / T, 15 / T, 20 / T$. As the derivatives with respect to the $\bar{z}$ coordinate in the source term suggest, thinner walls induce a larger baryon asymmetry. However, this effect is much more pronounced for the $H_{1}-H_{2}$ contribution.

For the $H_{1}+H_{2}$ contribution we find a baryon asymmetry which in the most favorable case is about 7 times larger than the observed value. Using kinetic momentum in the averaging, one obtains maximally (in the massless case) a further factor 2. As result, the assumption of maximal $\mathrm{CP}$ violation can only mildly be relaxed. Large complex phases are necessary, which are only compatible with EDM experiments if accidental cancellations happen or the first and second generation squarks have $\mathrm{TeV}$ scale masses. The $H_{1}-H_{2}$ contribution to $\eta$ is an order of magnitude larger, and smaller phases of the order $10^{-2}$ would be sufficient.

Let us finally compare our results with those of refs. [18, 23], which also started from the transport equations (5.23). The authors of ref. [18] also use the semiclassical method but consider only the $H_{1}+H_{2}$ contribution, and take somewhat different values for the interaction rates and diffusion constants. Our results are qualitatively similar. The dependence of the baryon asymmetry on $v_{w}$ shows small deviations. This may be due the fact, that in contrast to ref. [18] we exactly calculate the Greens function associated with the transport equation (5.23).

Ref. [23] is based on quantum transport equations. In this derivation of the chargino source terms also a $H_{1}-H_{2}$ contribution appears. The results in amplitude and velocity dependence are very similar to those presented here for the $H_{1}-H_{2}$ source. The small deviations in the regime of small $v_{w}$ may again be due to an approximation in the computation of the Greens function if ref. [23]. It remains to be seen if the $H_{1}-H_{2}$ contributions in the approach of refs. 212 23. and in the semi-classical method are related, and if they are indeed artificial as suggested by the use of kinetic momentum.

\footnotetext{
${ }^{4}$ Potential changes in $v_{w}$ due to reheating [26] were neglected.

${ }^{5}$ taking into account a numerical normalization error corrected in a forthcoming revised version of [18].
} 


\section{Conclusions}

In the first part we have given in some detail the procedure of solving numerically for the wall profile in the case of more then one Higgs field for a given effective potential. This extends a previous description [13]. Within this method we can also systematically search for CP violating solutions. It turns out - and this is confirmed in ref. [14] - that in the MSSM there is no indication of a spontaneous $\mathrm{CP}$ violation. However, in the larger parameter space of the NMSSM [28] such solutions can be found in 13. In the MSSM we remain with the possibility of explicit $\mathrm{CP}$ violating interactions, in particular of the charginos.

In the second part we treat transport around the stationary proceeding wall in the quasi-classical approximation. The solution of the set of Dirac equations for the charginos in the WKB approximation shows a split in the dispersion relations between particles and anti-particles at order $\hbar$ because of $\mathrm{CP}$ violation. We have written the full dispersion relations according to ref. [16] for canonical momenta, but also presented the relations for kinetic momenta. In ref. [18] arguments have been presented that one should use the kinetic momentum in Boltzmann transport equations. This would destroy all source terms of CP violation antisymmetric in the two Higgses which have been considered to be most important in older work on the MSSM. Also in order to compare with work in the spirit of quantum Boltzmann equations [21 23] we have kept open for both versions.

Of course one also has to test the two different types of momentum in averaging procedures, but this just gives a factor (about 2 ). Indeed, because $\tan \beta$ only varies very weakly in the wall, the contribution of the symmetric Higgs combination can not be neglected anyway. However, if the antisymmetric source is absent, rather large explicit $\mathrm{CP}$ violation is needed to obtain the observed baryon asymmetry even for favorable parameters of the MSSM. Thus the discussion of consistency with experimental EDM bounds due to accidental cancellations becomes relevant. On the other side this might point to an extension of the MSSM, e.g. to some version with a singlet. It is encouraging that we at least obtained a baryon asymmetry which is of the right order of magnitude compared to observations.

Acknowledgement We would like to thank M. Laine for collaboration in [13], technical support, and useful discussions. This work was partly supported by the TMR network Finite Temperature Phase Transitions in Particle Physics, EU contract no. FMRX-CT97-0122. S. H. is supported in part by the Alexander von 
Humboldt Foundation. P. J. is supported by the Deutsche Forschungsgemeinschaft.

\section{References}

[1] T. Asaka, K. Hamaguchi, M. Kawasaki and T. Yanagida, Phys. Rev. D61, 083512 (2000), hep-ph/9907559; W. Buchmüller and M. Plümacher, hep-ph/0007176.

[2] K. Kajantie, M. Laine, K. Rummukainen and M. Shaposhnikov, Phys. Rev. Lett. 77 (1996) 2887, hep-ph/9605288.

[3] D. Bödeker, P. John, M. Laine, and M. G. Schmidt, Nucl. Phys. B497 (1997) 387, hep-ph/9612364.

[4] B. de Carlos and J. R. Espinosa, Nucl. Phys. B503 (1997) 24, hep-ph/9703212.

[5] J. R. Espinosa, M. Quirós, and F. Zwirner, Phys. Lett. B307 (1993) 106, hep-ph/9303317; J. R. Espinosa, Nucl. Phys. B475 (1996) 273, hep-ph/9604320; M. Carena, M. Quirós, and C. E. M. Wagner, Nucl. Phys. B524 (1998) 3, hep-ph/9710401; M. Losada, Nucl. Phys. B537 (1999) 3, hep-ph/9806519; M. Losada, hep-ph/9905441; S. Davidson, T. Falk and M. Losada, Phys. Lett. B 463 (1999) 214, hep-ph/9907365; M. Laine and K. Rummukainen, Nucl. Phys. B535 (1998) 423, hep-lat/9804019; M. Laine and K. Rummukainen, Phys. Rev. Lett. 80 (1998) 5259, hep-ph/9804255.

[6] J. M. Cline and G. D. Moore, Phys. Rev. Lett. 81 (1998) 3315, hep-ph/9806354; J. M. Cline, hep-ph/9810267; J. M. Cline, hep-ph/9902328.

[7] J. M. Cline, G. D. Moore, and G. Servant, Phys. Rev. D60 105035,1999, hep-ph/9902220.

[8] N. Maekawa, Phys. Lett. B282 (1992) 387; A. Pomarol, Phys. Lett. B287 (1992) 331, hep-ph/9205247.

[9] J. R. Espinosa, J. M. Moreno, and M. Quirós, Phys. Lett. B319 (1993) 505, hep-ph/9308315. 
[10] J. M. Cline, K. Kainulainen, and A. P. Vischer, Phys. Rev. D54 (1996) 2451, hep-ph/9506284.

[11] K. Funakubo, A. Kakuto, S. Otsuki and F. Toyoda, Prog. Theor. Phys. 99 (1998) 1045, hep-ph/9802276; K. Funakubo, S. Otsuki and F. Toyoda, Prog. Theor. Phys. 102 (1999) 389, hep-ph/9903276.

[12] M. Laine and K. Rummukainen, Nucl. Phys. B545 (1999) 141, hep-ph/9811369; M. Laine and K. Rummukainen, Nucl. Phys. Proc. Suppl. 83 (2000) 577, hep-lat/9908045.

[13] S. J. Huber, P. John, M. Laine, and M. G. Schmidt, Phys. Lett. B475 104, hep-ph/9912278; P. John, Proc. of SEWM2000, hep-ph/0010277

[14] M. Laine and K. Rummukainen, hep-lat/0009025.

[15] A. G. Cohen, D. B. Kaplan, and A. E. Nelson, Ann. Rev. Nucl. Part. Sci. 43 (1993) 27, hep-ph/9302210.

[16] J. M. Cline, M. Joyce and K. Kainulainen, Phys. Lett. B417 (1998) 79, hep-ph/9708393.

[17] M. Joyce, T. Prokopec and N. Turok, Phys. Rev. D53, 2958 (1996), hep-ph/9410282.

[18] J.M. Cline, M. Joyce, K. Kainulainen, JHEP 0007 (2000) 018, hep-ph/0006119; K. Kainulainen, hep-ph/0003122; M.J. Cline, K. Kainulainen, Phys. Rev. Lett. 85 (2000) 5519, hep-ph/002272.

[19] G. Moore, JHEP 0003 (2000) 006, hep-ph/0001274.

[20] M. Joyce, K. Kainulainen and T. Prokopec, Phys. Lett. B474 (2000) 402, hep-ph/9910535.

[21] A. Riotto, Nucl. Phys. B518 (1998) 339, hep-ph/9712221; A. Riotto, Phys. Rev. D58 (1998) 95009, hep-ph/9803357.

[22] M. Carena, M. Quirós, A. Riotto, I. Vilja, C. E. M. Wagner, Nucl. Phys. B503 (1997) 387, hep-ph/9702409.

[23] M. Carena, J. M. Moreno, M. Quirós, M. Seco and C. E. Wagner, hep-ph/0011055. 
[24] G. D. Moore and T. Prokopec, Phys. Rev. D52 (1995) 7182, hep-ph/9506475; G. Moore and T. Prokopec, Phys. Rev. Lett. 75 (1995) 777 , hep-ph/9503296.

[25] P. John and M. G. Schmidt, hep-ph/0002050, revised version; P. John and M. G. Schmidt, Proc. of SEWM 2000, hep-ph/0012077.

[26] A. Mégevand, hep-ph/0011019.

[27] S. J. Huber and M. G. Schmidt, Eur. Phys. J. C10 (1999) 473, hep-ph/9809506; S. J. Huber, Proc. of SEWM '98, hep-ph/9902325; S. J. Huber and M. G. Schmidt, hep-ph/0011059; S. J. Huber, PhD thesis, Heidelberg, Oct. 1999.

[28] S. J. Huber, M. G. Schmidt, hep-ph/0003122 and forthcoming revised version.

[29] H. E. Haber, G. L. Kane, Phys. Rep. 117 (1985).

[30] M. Dine, R. G. Leigh, P. Huet, A. Linde, and D. Linde, Phys. Rev. D46 (1992) 550, hep-ph/9203203; M. Dine, R. G. Leigh, P. Huet, A. Linde, and D. Linde, Phys. Lett. B283 (1992) 319, hep-ph/9203201; A. D. Linde, Nucl. Phys. B216 (1983) 421.

[31] G. W. Anderson and L. J. Hall, Phys. Rev. D45 (1992) 2685.

[32] B.-H. Liu, L. McLerran, and N. Turok, Phys. Rev. D46 (1992) 2668.

[33] H. Kurki-Suonio and M. Laine, Phys. Rev. Lett. 77 (1996) 3951, hep-ph/9607382; J. Ignatius, K. Kajantie, H. Kurki-Suonio and M. Laine, Phys. Rev. D49 (1994) 3854, astro-ph/9309059; H. Kurki-Suonio and M. Laine, Phys. Rev. D54 (1996) 7163, hep-ph/9512202.

[34] J. M. Moreno, M. Quirós, and M. Seco, Nucl. Phys. B526 (1998) 489, hep-ph/9801272.

[35] J. M. Cline, J. R. Espinosa, G. D. Moore, and A. Riotto, Phys. Rev. D59 (1999) 065014, hep-ph/9810261.

[36] M. E. Carrington and J. I. Kapusta, Phys. Rev. D47 (1993) 5304; N. Turok, Phys. Rev. Lett. 68 (1992) 1803. 
[37] A. Kusenko, Phys. Lett. B358 (1995) 47, hep-ph/9506386; A. Kusenko, Phys. Lett. B358 (1995) 51, hep-ph/9504418.

[38] P. John, Phys. Lett. B452 (1999) 221, hep-ph/9810499; P. John, in Proc. of SEWM 98, hep-ph/9901326; P. John, PhD thesis, Heidelberg, 1999.

[39] W. Press, Numerical Recipes in C, Cambridge University Press, 2 ed., 1991.

[40] D. Knuth, The Art Of Computer Programming, vol. 2, Addison Wesley, 2 ed., 1981; M. Abramowitz and I. A. Stegun, Handbook of Mathematical Functions with Formulas, Graphs and Mathematical Tables, John Wiley \& Sons, 1972.

[41] H. J. Rothe, Lattice Gauge Theory, World Scientific, 2 ed., 1997.

[42] M. G. Perez and P. van Baal, Nucl. Phys. B429 (1994) 451, hep-lat/9403026.

[43] P. Huet and A.E. Nelson, Phys. Rev. D53 (1996) 4578, hep-ph/9506477.

[44] M. Aoki, A. Sugamoto and N. Oshimo, Prog. Theor. Phys. 98 (1997) 1325, hep-ph/9706287.

[45] N. Rius and V. Sanz, Nucl. Phys. B570(2000) 155, hep-ph/9907460.

[46] J. Ellis, S. Ferrara and D.V. Nanopoulos, Phys. Lett. B 114 (1982) 231; W. Buchmüller and D. Wyler, Phys. Lett. B 121(1983) 321; J. Polchinski and M.B. Wise, Phys. Lett. B 125 (1983) 393.

[47] Y. Kizukuri and N. Oshimo, Phys. Rev. D 46 (1992) 3025.

[48] A. Pilaftsis and C. E. M. Wagner, hep-ph/9902371.

[49] Y. Kizukuri and N. Oshimo, Phys. Rev. D46 (1992) 3025; T. Ibrahim and P. Nath, Phys. Lett. B418 (1998) 98, hep-ph/9707409; M. Brhlik, G. Good, and G. L. Kane, Phys. Rev. D59 (1999) 115004, hep-ph/9810457; D. Chang, W.-Y. Keung, and A. Pilaftsis, Phys. Rev. Lett. 82 (1999) 900, hep-ph/9811202; T. Falk and K. A. Olive, Phys. Lett. B439 (1998) 71, hep-ph/9806236; T. Falk, K. A. Olive, and M. Srednicki, Phys. Lett. B354 (1995) 99, hep-ph/9502401.

[50] D. Comelli and M. Pietroni, Phys. Lett. B306 (1993) 67, hep-ph/9302207. 
[51] D. Comelli, M. Pietroni and A. Riotto, Nucl. Phys. B412 (1994) 441, hep-ph/9406368.

[52] L. McLerran, M. Shaposhnikov, N. Turok and M. Voloshin, Phys. Lett. B256 (1991) 451; A.E. Nelson, D.B. Kaplan and A.G. Cohen, Nucl. Phys. B373 (1992) 453;

[53] T.D. Lee, Phys. Rev. D8 (1973) 1226.

[54] K. Olive, astro-ph/9901231.

[55] G. F. Giudice, M. Shaposhnikov, Phys. Lett. B326 (1994) 118, hep-ph/9311367. 